\title{
Avant-gardes et expérimentations des formes dans les revues pharmaceutiques suisses: le cas Sandorama (1962-1965)
}

Muriel Pic

\section{Résumé}

Cet article fait état de l'expérimentation formelle (littéraire, graphique et cinématographique) dans les revues pharmaceutiques suisses des années Soixante à partir d'une étude de cas: la revue Sandorama des Laboratoires Sandoz. Il envisage le rapport entre arts, médecine et commerce en montrant que la mise en confiance du public des médecins lecteurs de la revue s'opère par la voie des formes. L'inventivité de ces dernières s'inscrit dans le processus plus global d'une réorganisation du marketing pharmaceutique après la Seconde guerre mondiale en raison notamment de l'arrivée des médicaments psychotropes sur le marché.

Mots-clés: revues pharmaceutiques, arts, littérature, commerce, graphisme, cinéma, montage

\section{Summary}

This article reports on formal experimentation (literary, graphic and cinematographic) in Swiss pharmaceutical journals in the 1960s based on a case study: the Sandorama journal of Laboratoires Sandoz. It looks at the relationship between arts, medicine and commerce, showing that the public trust of the doctors who read the journal is built up through forms. The inventiveness of the latter is part of the more global process of a reorganization of

Muriel Pic, Prof. FNS, Université de Berne, Institut de langue et littérature françaises, Länggassstrasse 49, 3012 Berne, Tel: 0316314506 / 07894696 76, muriel.pic@rom.unibe.ch 
pharmaceutical marketing after the Second World War, due in particular to the arrival of psychotropic drugs on the market.

Keywords: pharmaceutical reviews, arts, literature, commerce, graphic design, cinema, montage

En ouvrant une revue pharmaceutique suisse des années Soixante, le lecteur est frappé par la qualité esthétique de son contenu et le caractère expérimental de ses dispositifs formels. Certaines n'ont rien à envier aux objets d'art avant-gardistes et sont déjà prisées des collectionneurs (souvent des médecins attentifs à la création de manière générale). Ces revues, qui représentent un corpus vaste et plurilingue, émanent des Laboratoires Geigy, Ciba, La Roche, Sandoz. Ce sont, hors bulletins et brochures, Documenta Geigy, Symposium Ciba, Hexagone pour La Roche, Panorama/Sandorama pour Sandoz. Internationalement diffusées dans les filiales (une soixantaine en 1966 pour Sandoz), elles sont traduites en français, anglais, espagnol, italien, et témoignent de l'importante réorganisation des laboratoires pharmaceutiques en matière de commercialisation et de publicité après la Seconde guerre mondiale. ${ }^{1}$ Dans ce cadre, les arts occupent une place fondamentale les firmes employant au sein des nouveaux services de «propagande», selon le terme alors consacré, des désigneurs, des dessinateurs, des cinéastes, des écrivains, et font appel aussi à des contributeurs extérieurs. L'envergure des campagnes publicitaires donnent aux arts un statut nouveau dans le monde médical: ils ne sont plus seulement les vecteurs d'une humanisation et d'une esthétisation de la science, mais les acteurs de son économie.

Toutefois, si la publicité devient une question de premier ordre pour l'industrie pharmaceutique suisse dans les années Soixante, c'est également en raison de l'émergence d'un nouveau marché prometteur: la psychopharmacologie. Le développement des médicaments psychotropes stimule l'inventivité formelle à une époque où la science est devenue attentive aux phénomènes de la perception. Un «Geigy style» s'affirme alors, vite synonyme d'un «Swiss style», qui désigne une école de graphisme qui va marquer l'histoire de cette discipline artistique. Car si les artistes se plient aux exigences de la propagande (véhiculer l'image de marque et faire de la publicité pour les médicaments), c'est en innovant librement. À partir des contraintes données par les laboratoires, ils varient sur les représentations de la maladie, du soin, du corps (fig. 1 et 2.), chargés de promouvoir des produits pharmaceutiques en suscitant la confiance des prescripteurs et des consommateurs. Je me propose

1 Gaudillière 2015, 169-170.

Gesnerus 77 (2020) 


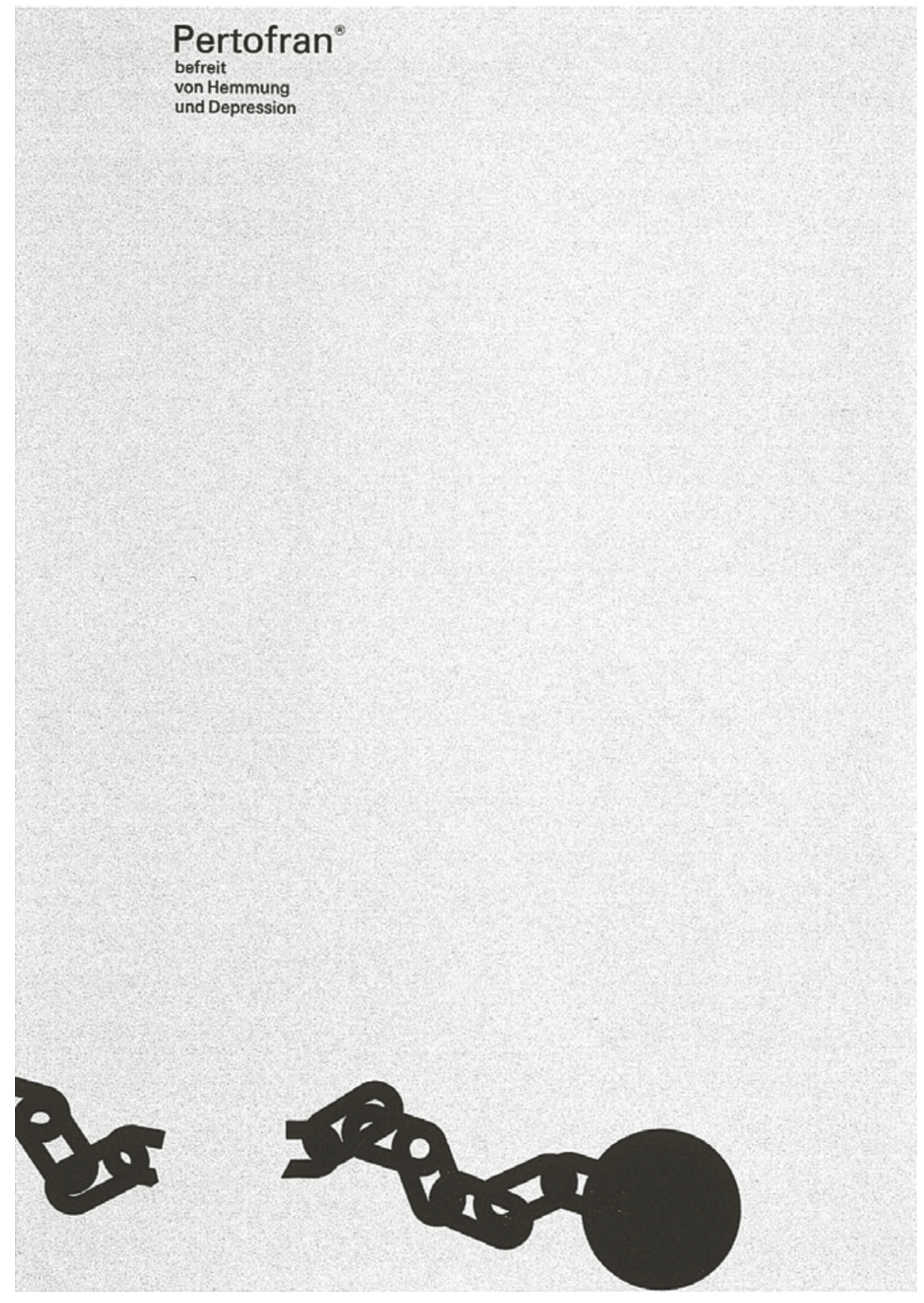

Fig. 1. Max Schmid, Pertofran libère de l'inhibition et de la dépression, vers 1962. Offset, $29.6 \times 21 \mathrm{~cm}$.

66 Gesnerus $77(2020)$ 


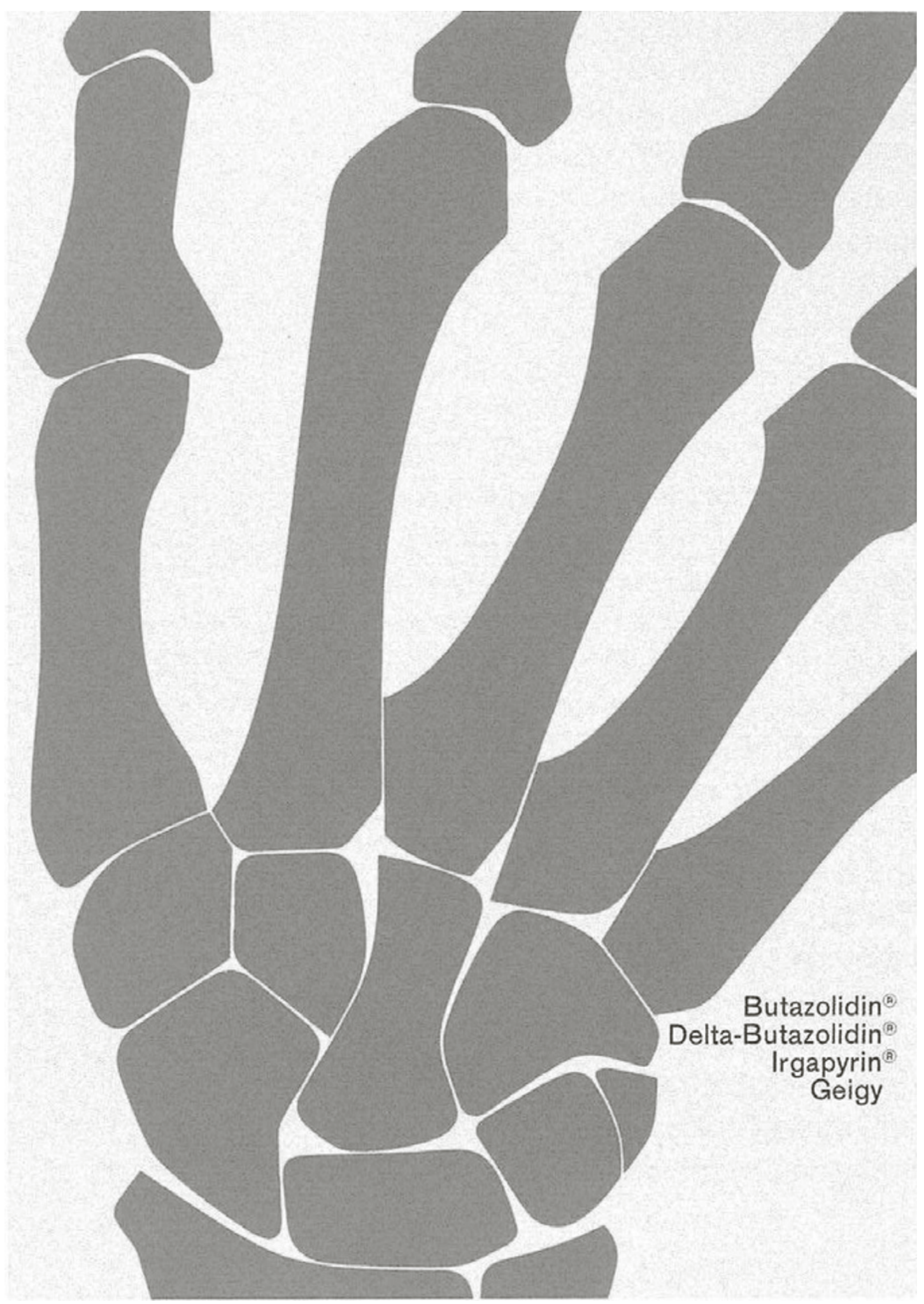

Fig. 2. Roland Aeschlimann, Butazolidin/Delta-Butazolidin/Irgapyrin/Geigy, CH/ $\mathrm{CH}$, avant 1963. Offset, $21 \times 14.7 \mathrm{~cm}$. 
donc de voir comment se nouent, par le travail des formes, arts, commerce et médecine, dans les revues pharmaceutiques suisses des années Soixante, et cela, à partir d'une étude de cas: la première série de livraisons de la revue Sandorama des Laboratoires Sandoz entre 1962 et 1965.

\section{La revue pharmaceutique: un objet humaniste entre histoire médicale et littéraire}

Avant d'ouvrir Sandorama, il faut rappeler que l'objet revue, en l'occurrence pharmaceutique, est inscrit dans l'histoire de l'humanisme depuis le XVIII ${ }^{e}$ siècle au moins, et il faut ici donner quelques points de repère historiques, même insuffisants. De manière générale, «la revue est relativement aisée à définir, parce que la bibliothéconomie internationale a forgé les termes d'un consensus à peu près stabilisé», bien que sa réalité soit passablement complexe:

La revue (de l'anglais review) est une publication périodique au contenu variable. Sa périodicité la distingue du livre ou de la brochure. Son sens est à peu près celui du mot «journal», qui s'est, lui, spécialisé dans l'acception «journal quotidien», que l'on appelait auparavant la «gazette». [...] Souvent illustrée, elle s'offre dans un format et une typographie qui ne sont pas le fait du hasard; elle comporte de l'éditorial, de la publicité, des annonces littéraires ou non (ainsi de la publicité «par sympathie»), des couvertures, des tables (souvent erronées), des mentions d'éditeur responsable, de comité de rédaction (souvent factices), des adresses, des bulletins d'abonnement ou des feuillets séparés destinés au lectorat. ${ }^{2}$

Autrement nommée journal, gazette, magazine, le terme revue a pu aussi se spécifier au $\mathrm{XX}^{\mathrm{e}}$ siècle pour désigner un support qui véhicule moins du divertissement culturel et de l'information grand public que des connaissances scientifiques. L'hétérogénéité des supports, des formes, des connaissances mobilisées, la grande flexibilité d'invention des normes de lisibilité et de visibilité font de la revue un objet extrêmement intéressant pour saisir des réalités historiques complexes, comme l'hétérogénéité socio-culturelle des acteurs d'un mouvement qu'elle regroupe et fait exister.

Les revues naissent en Europe à l'époque des Lumières pour fédérer des groupes et ouvrir des espaces d'échanges entre des esprits formés tout aussi bien à la philologie humaniste qu'aux sciences naturelles. En France, Le Journal des savants est créé en 1665; en Allemagne, autre pays de l'Aufklärung triomphant, les revues publient des lettres ouvertes, des recensions critiques, des essais. Au XVIII ${ }^{\mathrm{e}}$ siècle, certaines revues deviennent incontournables pour la vie publique de la pensée, par exemple le journal berlinois de Friedrich Nicolai et de Moses Mendelssohn, Briefe, die Neueste Literatur betreffend; ou

2 Aron/Denis 2008, 2. 
encore, le fameux Athenaeum des premiers romantiques, les frères Schlegel et Novalis. Au XIX ${ }^{\mathrm{e}}$ siècle, dans les domaines désormais distincts des sciences et des lettres, les revues fleurissent: le positivisme rend compte des progrès scientifiques, le naturalisme publie les romans en feuilletons; les écrivains s'inspirent de la presse scientifique et médicale - que l'on pense seulement au Horla de Maupassant -, tandis que la poésie scientifique abonde dans les journaux pharmaceutiques, parfois liée à la publicité qui manie pour l'occasion la versification et la rime. ${ }^{3}$ Retraçant l'émergence des revues scientifiques en France et leur inflation jusqu'au XIX ${ }^{\mathrm{e}}$ siècle, Hugues Marchal dresse un bilan qui insiste sur la progressive séparation du domaine scientifique et littéraire «selon un processus que Bonald présente vers 1807 comme une guerre civile qui met fin à leur confédération antérieure»:

La revue scientifique moderne prend alors forme par sa capacité à se spécialiser, pour chasser la vie littéraire de ses pages. Dans les années 1820 et 1830, les sociétés savantes se dotent de bulletins réguliers, tels les Mémoires de l'Académie des sciences, dont Arago impose la publication en 1835, et les titres se multiplient au point que des outils d'inventaire doivent être créés: L'Année biologique, fondée en 1897, propose chaque année un résumé des principaux «travaux de biologie», tandis qu'en 1909, Romans-Revue, guide de lectures pour le public catholique, constate: «Nous ne pouvons songer ici à faire le dénombrement de tous les périodiques scientifiques, même uniquement français, et encore moins à les passer en revue.» Dans ce contexte de forte croissance de la matière savante, l'actualité littéraire et l'actualité scientifique s'énoncent dans des organes distincts, et cette répartition est illustrée par la création de la prestigieuse Revue des cours scientifiques, puis Revue scientifique ou «revue rose», éditée à partir de 1863 chez Germer Baillière. ${ }^{4}$

$\mathrm{Au} \mathrm{XX}^{\mathrm{e}}$ siècle, on assiste à une explosion des parutions périodiques avec deux moments distincts: avant et après la Seconde Guerre mondiale. Durant la première moitié du siècle apparaissent en France des revues médico-littéraires qui cherchent à promouvoir un humanisme scientifique fondé sur l'adéquation platonicienne du beau et du bien. Martina Diaz note bien: «Étant donné que la plupart de leurs collaborateurs ont exercé sur le front, ces revues semblent poursuivre une aspiration humaniste, proposant une vision idéalisée de la figure du médecin, qui allierait expertise technique et culture lettrée.. ${ }^{5}$ L'illustration de ces revues (Epidaure, La Flamme, Art et médecine, L'Archer, L'Esprit médical) contraste fortement avec celle des revues artistiques et littéraires avant-gardistes où la photographie et le collage priment, les formes exprimant un engagement politique anarchiste et/ou révolutionnaire. À l'inverse, les revues médico-littéraires reproduisent surtout des œuvres peintes et des sculptures, la répartition des textes et des images se pliant au protocole illustratif traditionnel qui met la seconde au service du

3 Marchal 2013, 2018; Aron 2018; Guellec/Hache-Bissette 2012; Guellec 2010, 2017; Suter 2010, 2016.

4 Marchal 2015, 40.

5 Diaz 2018, 15. 
premier. De manière générale, les arts semblent être là surtout pour rendre plus humain un médecin qui, sous le règne du positivisme, a perdu sa capacité d'émotion. Ces revues, s'apparentant à des magazines mondains réservés au corps médical, ${ }^{6}$ sont dirigées par des médecins amateurs de littérature et d'arts, sensibles à la vie esthétique, et qui font appel à des écrivains de renom comme Paul Valéry. La plus importante de ces revues, qui connaît son âge d'or de 1930 à 1936, est sans aucun doute Art et Médecine du Professeur François Debat, devenue une vitrine publicitaire pour le laboratoire éponyme. Martina Diaz en décrit bien le fonctionnement:

La principale tentative de réunir médecins et écrivains à l'Entre-deux-guerres est le fait du Dr François Debat, qui édite Art et médecine à Paris de 1929 à 1939, dont le sous-titre est «revue mensuelle réservée au corps médical». Rebaptisée épisodiquement La Revue du médecin (en 1929-1930 et entre 1936 et 1938), Art et médecine connaît ses années de gloire entre 1930 et 1936: elle se distingue alors clairement de ses concurrentes contemporaines par la qualité de son impression, la qualité de ses iconographies, et la cohérence de ses numéros mensuels, toujours consacrés à un thème ou à une région française ou coloniale - car elle «ne publie généralement, que des choses de France» (avr. 1935, 11). Art et médecine accueille en vérité un défilé des écrivains et des médecins les plus importants de cette décennie (Maurois, Duhamel, Barrès, Durtain, Bourget, Cocteau, Romains, Morand, Henri Mondor, Dorgelès, Louis Dartigues, les frères Faure, etc). Ces plumes prestigieuses côtoient des collaborateurs réguliers, qui tiennent des rubriques littéraires, théâtrales, musicales ou d'opinion. ${ }^{7}$

L'humanisme conservateur de ce type de revues, qui esthétise la médecine par les arts réduits au statut d'instruments, s'incarne pour Art et Médecine dans la figure du Professeur Henri Mondor, idéal du «médecin littérateur».8 La réalité politique de la revue, immédiatement perceptible dans les dispositifs formels, n'étonne guère: elle devient pétainiste en 1940, Debat montrant ses sympathies pour le fascisme déjà à la fin des années Trente. ${ }^{9}$

Après la Seconde Guerre mondiale, force est de constater que le développement des revues pharmaceutiques est au contraire caractérisé par l'innovation formelle, comme nous allons le voir à présent à partir du cas Sandorama. Comme tous les journaux et revues pharmaceutiques, cette dernière véhicule l'image de marque du laboratoire et de ses produits, en recourant stratégiquement aux arts: ces derniers donnent du crédit à un laboratoire pharmaceutique montrant ainsi l'importance qu'il accorde aux valeurs humanistes (et non pas seulement scientifiques et économiques). ${ }^{10}$ Toutefois, il ne s'agit là que d'un aspect mineur du rôle des arts et de la littérature dans Sandorama, qui se distingue plutôt par ce que l'on peut nommer un humanisme expérimental laissant une grande liberté à l'expression formelle dans ses colonnes, cher-

6 Diaz 2017.

7 Diaz 2018.

8 Diaz/Wenger 2018.

9 Diaz 2018, 26.

10 Neuburg 1967. 
chant l'innovation dans ce domaine comme dans celui de la science. Cela ne va pas sans un contrôle attentif par le département de la propagande à Bâle, tant en ce qui concerne la qualité des publications internationales que la surveillance de leurs contenus pour l'image de marque de la firme. Si les seules archives administratives conservées dans le fonds Sandoz à Novartis entre le siège bâlois et la succursale parisienne (dirigée par le Dr Lantiez) ne couvrent pas la période concernée par la parution de Sandorama, mais les années immédiatement précédentes (1956-1960), elles permettent néanmoins de voir comment tout est passé au peigne fin par Sandoz S.A. des rencontres entre scientifiques aux études de marché en passant par les envois de documentation, les observations sur la place du logo de la firme dans une brochure ou le style employé pour décrire les effets d'un médicament. Les revues n'échappent bien entendu pas à ce contrôle, d'autant que chargées d'informer, divertir et vendre, elles doivent donner forme à la maladie, la médication, la guérison. Dans ce processus de mise en représentation s'opère nécessairement une mise à distance vis-à-vis de la réalité médicale. L'intervention d'artistes dans la réalisation de la publicité comme dans la rédaction d'articles et la production de dessins implique en effet qu'un regard extérieur soit posé sur la communauté médicale. La vigilance est donc de rigueur, mais il est évident que l'heure n'est pas à la restriction de l'inventivité formelle, le courant de la révolution psychopharmacologique ouvrant une nouvelle ère des soins psychiatriques et des expérimentations liées à la perception.

En octobre 1959, Sandoz lance une enquête pour savoir combien de personnes ouvrent le journal Triangle, étude qui conduira à l'édition d'une nouvelle revue, plus divertissante et moins scientifique, à savoir Panorama. Cette dernière aboutira rapidement à une version francophone, destinée à la France, la Suisse romande et la Belgique: Sandorama. Un courrier du 23 décembre 1960 date ce projet et signe l'acte de naissance de la revue. Adressé au Dr Lantiez qui dirige la filiale parisienne par la maison mère de Bâle (qui signe systématiquement «Sandoz S.A.» sans que l'on puisse savoir qui se charge de ces courriers), il accompagne l'envoi, sous pli séparé, des «3 premiers numéros en allemand de notre nouvelle revue Panorama en vous priant de bien vouloir examiner comment une édition française pourrait se réaliser», cette dernière devant également compter 16 pages et adopter un format plus réduit de $31 \times 44 \mathrm{~cm}$. Elle devra être imprimée en France afin «de tenir compte de nécessités locales (autres formes, autres noms, etc.) [...] La traduction, la rédaction et la création d'articles nouveaux en français se fera à Bâle en pleine collaboration avec vous. $»^{11}$

11 Archives Novartis. M 320.071 1-14 (1956-1960).

Gesnerus 77 (2020) 


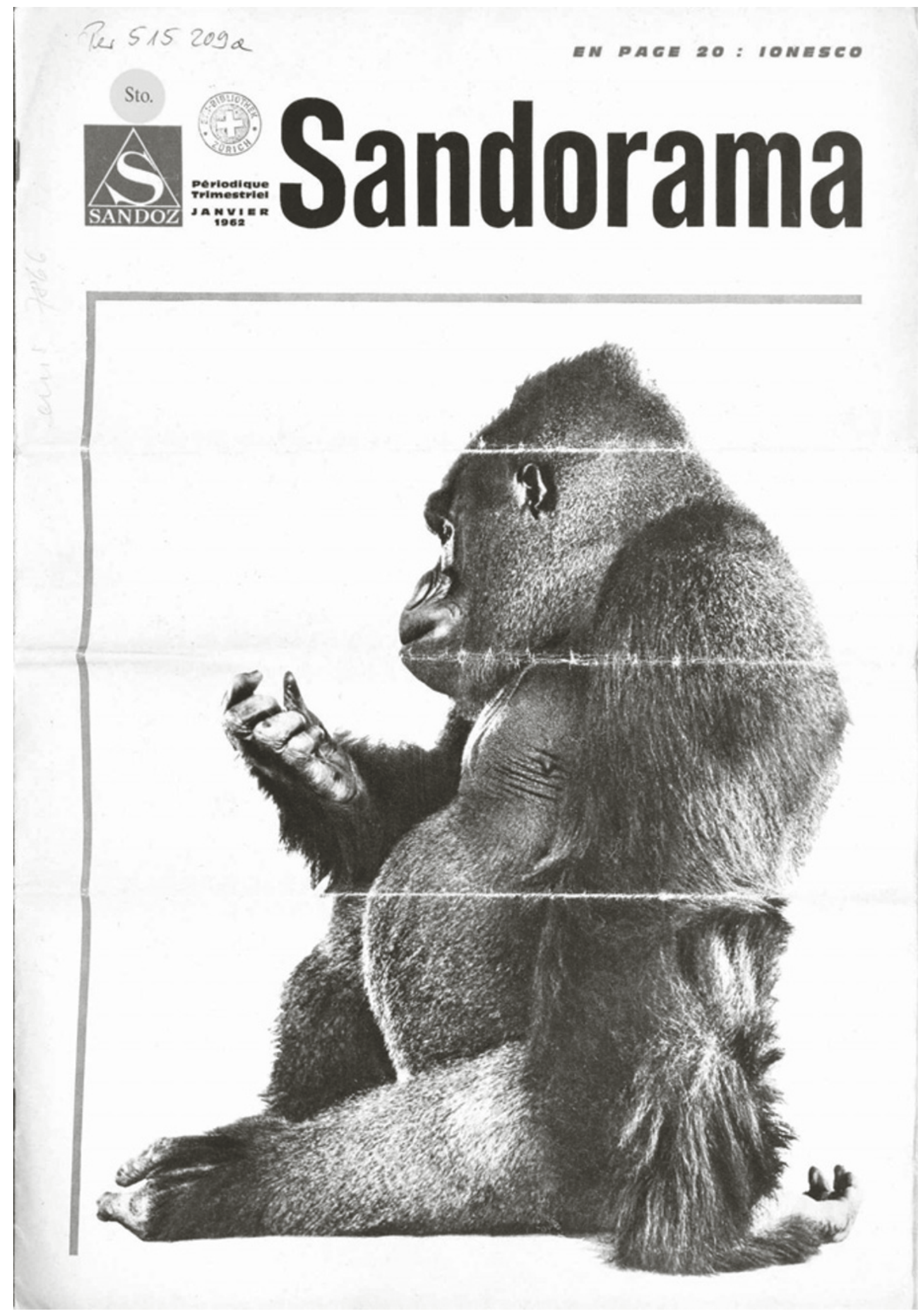

Fig. 3. Première de couverture de Sandorama (automne 1963). 


\section{L'ARCHE DE NOÉ}

(VESTIBULE DE SAINT MARC)

par MICHEL BUTOR

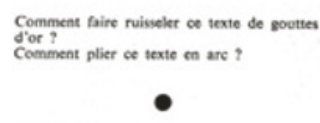

Yoidi maintenant rare du Delluger.
Do obte de la place :

DIXIT DOMINUS AD NOE FAC TIBI

Dieu dit. $\mathrm{A}$ Not : a ... Fais-teil une arche en

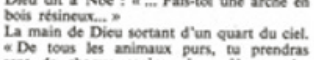

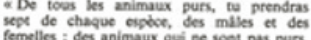

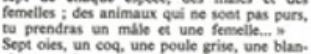

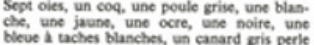

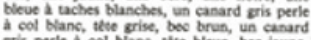

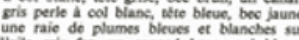

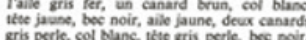

gris perte, col slunc, itte gris perle, bee soir.

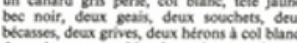

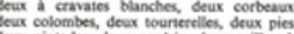

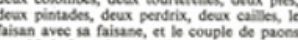

ue Not vient dans ses bras.

Deux serpents enlackes, deux ours impatients
deux renarts aboyant, deux leogards flairan,

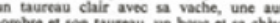

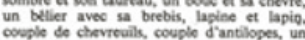

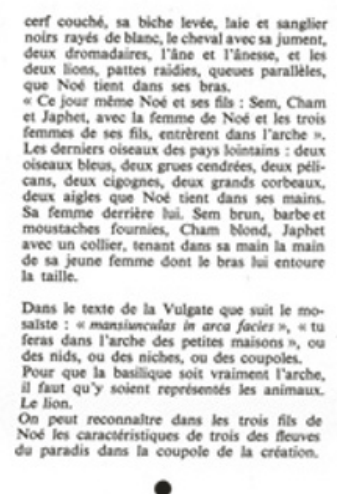

Du coté de l'église

FACTUM EST DILUVIUM QUADRA.

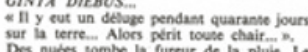

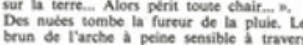

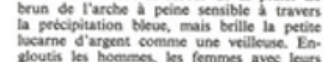

bijour, un entant, un dromedadire, un uav.

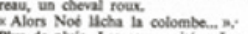

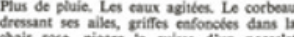

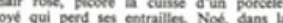
crande festetre ouverte, elikve à deux main bout de nes ailies avant de prendre son vol.

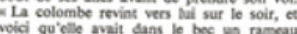
d'oliviet

Comme it laceseille !
Jo mets mon are dass la mobe, et il deviendra Eut

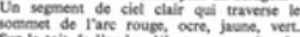
Sart le toit de liarches stebartent une pintade

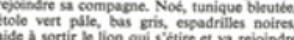
Wuster mosteau bleu agrafes sur sa tunique masue a bordure S'or, bas verts: Cham, mameas carnts 4 or, bordte d'or, bas pourpers

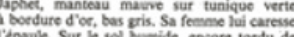
Esves, un arbre mor. erf et a bicte, l'ours et l'ourie ; dewe

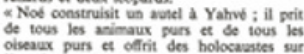
quil $a$ is metme forme que celui d'Abel drique ef quit ent protege par une petite

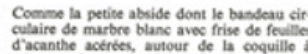
minces rainures mord sur la mosalyse. Bulsons asisant des caux, des fles rases. Sauves ceux qui foet des navires.

Les lifes rouges cernent les restaurations

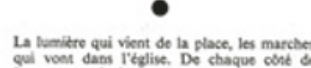

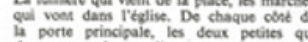

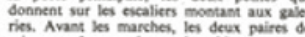

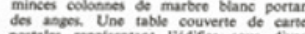

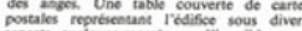

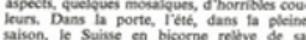

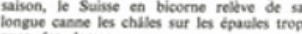
Au dessus, une mince vierge Here dans sa parmi les plas anciennes de ta basilique. Au-dessus, danst in poneque, un Saint Marc alement levece, les bras etendus, le cores en $\mathrm{S}$.

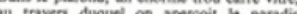

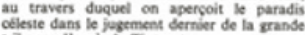

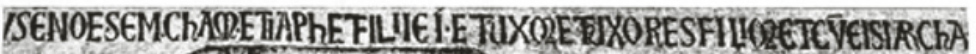

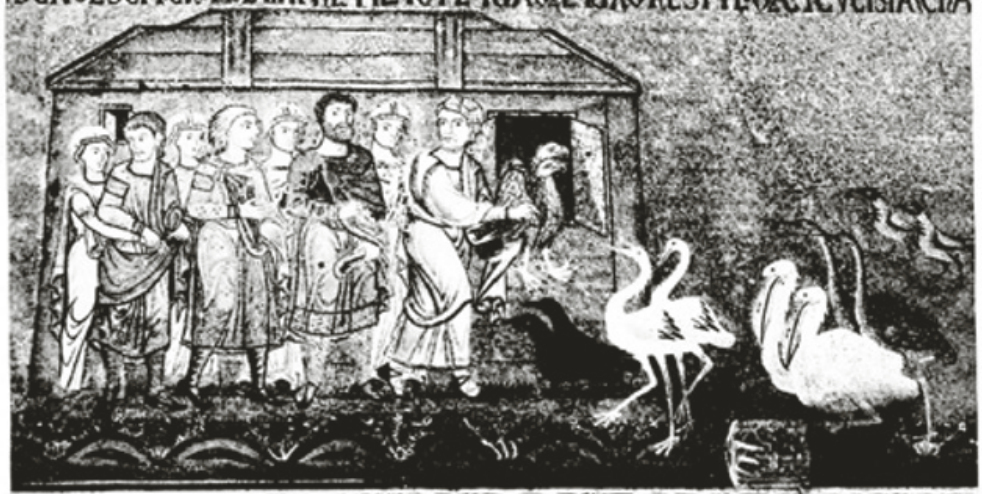

Fan

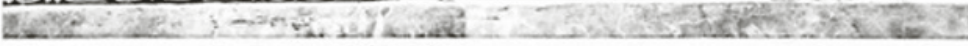

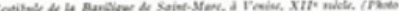

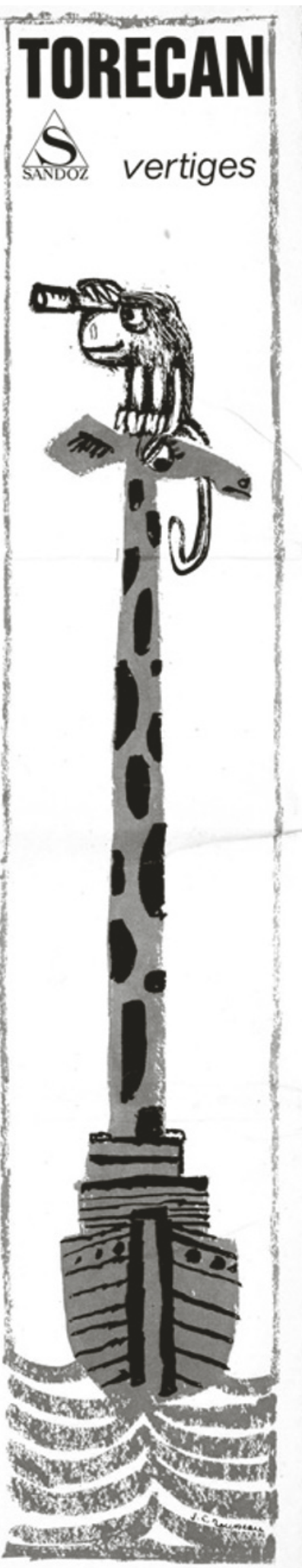

Fig. 4. Dernière de couverture de Sandorama (automne 1963). 
Le premier numéro de Sandorama (fig. 3 et 4) paraîtra en avril 1962 en l'espèce d'un périodique trimestriel des Laboratoires Sandoz «strictement réservé au personnel médical» (comme la plupart des revues pharmaceutiques) et émanant du «département de la propagande ». Le dernier numéro de Sandorama, paru en allemand en avril 1994, nous donne aussi quelques indications sur la genèse de la revue, dont la dénomination a été généralisée à plusieurs parutions étrangères. Un article de Peter Lattmann nous apprend que la revue est née d'une initiative du Professeur Josef Neugebauer qui dirigeait alors le département de l'Information médicale des Laboratoires Sandoz. Curieusement, l'auteur ne pas fait mention de la parution francophone de Sandorama, dont on constate qu'elle s'est relativement autonomisée par rapport à l'homologue germanophone de Bâle, contrairement à ses consœurs anglaises, espagnoles, italiennes. Sandorama France n'est en effet pas une stricte traduction de la revue bâloise. Finalement conçue à Paris où elle est imprimée chez Les Fils de Victor-Michel, dont la renommée dans la presse graphique remonte à la fin du $\mathrm{XX}^{\mathrm{e}}$ siècle, la revue reprend parfois certains articles à Panorama. Cette dernière publie par exemple en décembre 1962 un article sur l'almanach des expressionnistes allemands, Le Cavalier bleu (1912), signé par l'historien de l'art Pierre Cabane dans Sandorama en octobre de 1962. En revanche, les publicités des médicaments sont identiques entre les deux journaux qui, de surcroît, partagent un ascendant psychopharmacologique. Le premier numéro de juin 1960, publié sous le titre Das ärztliche Panorama - eine Sandoz-Zeitschrift, donne d'ailleurs en couverture la photographie d'une culture de psilocybine (fig. 5). Les vertus thérapeutiques de ce champignon psychotrope sont alors expérimentées aux Laboratoires Sandoz à Bâle par Albert Hofmann, qui a découvert ce psychotrope grâce au Professeur Roger Heim, mycologue et directeur du Musée d'Histoire Naturelle. Ce dernier envoie pour la première fois un échantillon du champignon à Sandoz en 1956, daté par un courrier entre la filiale parisienne et la maison mère du 30 novembre 1956.

C'est donc sous le signe de la psychopharmacologie que naissent Panorama et Sandorama, cette dernière conservant un tropisme psychotrope et psychiatrique à plusieurs niveaux: d'une part, dans les publicités pour des médicaments psychotropes ou des analgésiques puissants (Melleril, Torécan, Bellergal, Deseril, Optalidon, Palerol), conjointement à des anti-allergiques (Sandostène), des régulateurs du rythme cardiaque (Cédilanide), des comprimés de Calcium. D'autre part, dans les contenus, liés à la psychiatrie ou à la perception des formes, avec un éventail d'approches extrêmement large: de l'irrationnel dans l'art à l'art-thérapie et aux résumés de séances du Congrès du «Collegium Internationale Neuropsychopharmacologicum». Enfin, dans l'usage immodéré des 

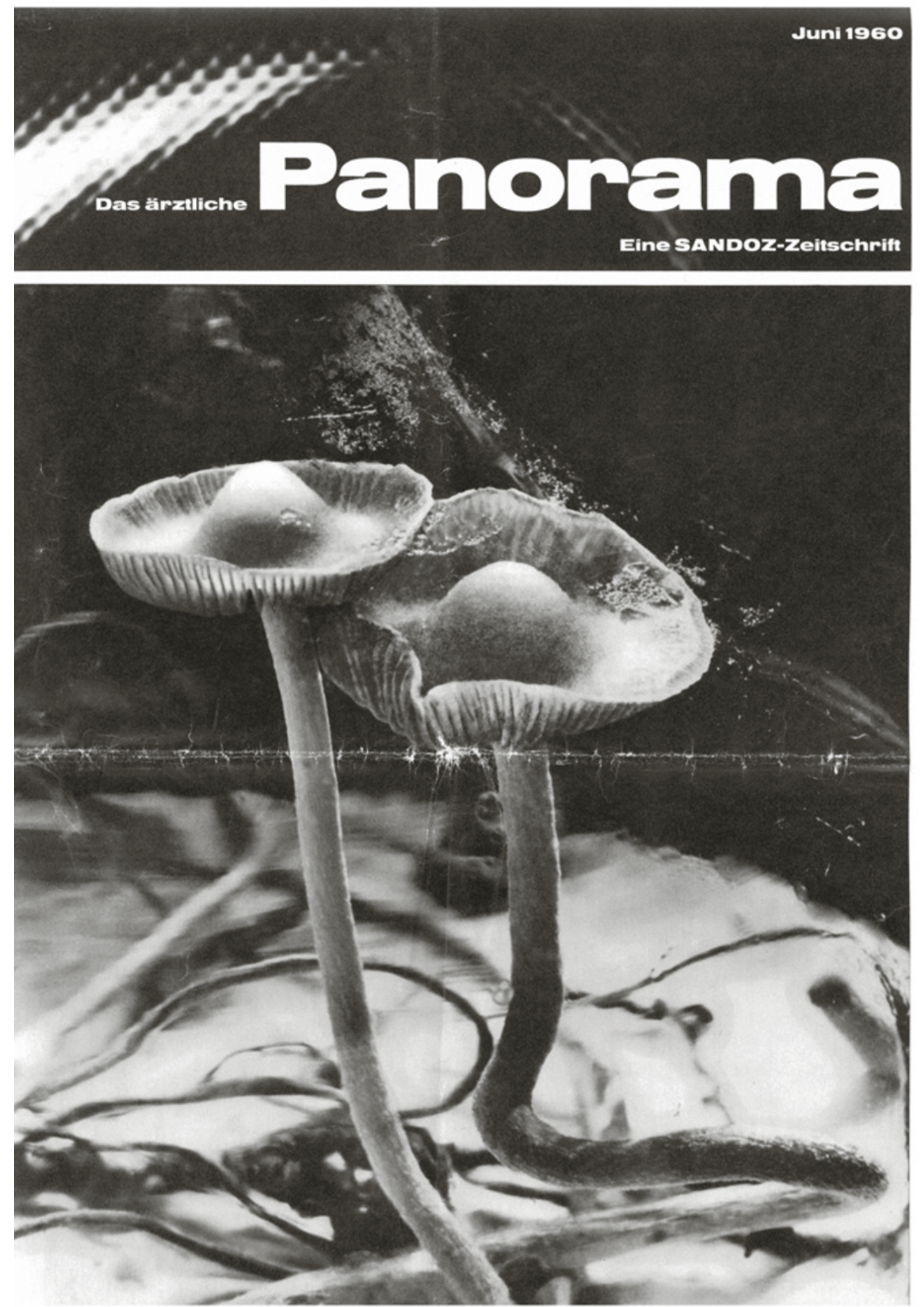

Fig. 5. Couverture du premier numéro de Panorama (1960). 
formes graphiques et des images, et des choix d'articles sur la science et sur les arts dont l'optique commune est celle d'une expérimentation de la perception.

Il faut rappeler brièvement ici que les recherches de la chimie, la botanique, l'ethnographie et la psychiatrie sur les possibilités thérapeutiques des psychotropes (mescaline, LSD25 et psilocybine) ont commencé au début du XX $\mathrm{X}^{\mathrm{e}}$ siècle et ont été particulièrement bien développées à Paris dans les années Cinquante et Soixante, ce qui explique aussi peut-être en partie la marge de manœuvre accordée à Sandorama. Dès 1950, les institutions psychiatriques parisiennes, en particulier l'hôpital Sainte-Anne, collaborent avec le laboratoire pharmaceutique bâlois. ${ }^{12}$ L'un des principaux interlocuteurs de Hofmann est le Professeur Jean Delay qui enseigne à la faculté de médecine et exerce à la Clinique des maladies mentales et de l'encéphale à Saint-Anne, où se déroulent la plupart des expérimentations. «Delay et collaborateurs», comme Sandoz S.A. les désigne, mènent leurs recherches sur la mescaline et le LSD 25, dont les molécules ont été isolées avant et pendant la Seconde Guerre mondiale, puis à partir de 1958 sur la psilocybine. En 1957, Delay et Pierre Deniker listent les propriétés des neuroleptiques: «Les psychoanaleptiques ou stimulants psychiques, avec les neuroleptiques ou stimulants de la vigilance, et les thymoanaleptiques ou stimulants de l'humeur ou antidépresseurs; les psycholeptiques ou sédatifs psychiques avec les hypnotiques, les anxiolytiques ou tranquillisants, et les neuroleptiques». Enfin, les psychodysleptiques ou les hallucinogènes qui perturbent l'activité mentale et n'ont pas d'usage thérapeutique. ${ }^{13}$ Telle sera en effet la conclusion de cette classification de Delay et Deniker, qui vient après celle de Louis Lewin en 1927, concernant les psychodysleptiques. Les recherches menées sur les psychotropes se traduisent par des expérimentations, dont l'enjeu est de retranscrire le vécu de l'intoxication. Elles touchent empiriquement à la perception avec la description des hallucinations et des troubles cénesthésiques, et impliquent une grande attention aux formes. Les sujets des expérimentations sont soit les médecins qui se livrent à l'auto-observation, soit les aliénés qui sont observés et invités à traduire plastiquement les effets des psychotropes, ou encore des volontaires, bien souvent des artistes. Jusqu'en 1959, Sandoz distribue gratuitement pour des recherches thérapeutiques justifiées des échantillons dans le monde entier, puis vendra le Delysid (ou LSD-25) aux professionnels. Le produit, exclusivement destiné aux psychoses expérimentales, sera retiré du marché en 1966, le laboratoire perdant le contrôle sur les usages hors cadre scientifique de ce dernier avec la vague psychédélique nord-américaine. 
La première série de Sandorama, revue née dans le contexte des recherches sur la thérapeutique des psychotropes, témoigne de l'importance de l'invention formelle à cette époque où les troubles de la perception sont au centre des attentions. Distincte du psychédélisme, tant dans ses ambitions littéraires que graphiques, Sandorama résulte des échanges entre Bâle et Paris. Elle traduit visuellement son identité franco-helvète en alliant au graphisme publicitaire de la pharmaceutique suisse des éléments culturels propres à la capitale française (littéraires, plastiques et cinématographiques) et une information médicale pertinente à l'échelle mondiale qui donne sa préférence aux questions relevant de la psychiatrie (où elle trouve certainement son plus gros contingent de lecteurs) ou à des questions d'imaginaire et de perception, domaines explorés par la psychopharmacologie. Si Sandorama ouvre largement ses colonnes aux arts, ce n'est donc pas seulement pour se donner du crédit, mais pour laisser une place à l'expérimentation formelle conjointement à l'expérimentation scientifique.

\section{L'expérimentation des formes littéraires et visuelles: pour une communauté Sandorama}

La première série de la revue Sandorama comprend 12 numéros: 1962 (janvier, avril, juillet, octobre); 1963 (janvier, avril, été, automne); hiver 1963-1964; 1964 (printemps, automne); 1965 (mars). On constate qu'à partir de 1963, la désignation des périodes change, et qu'en 1964, seuls trois numéros paraissent et un seul en mars 1965. La revue cherche constamment la meilleure formule. Dès janvier 1962, chaque numéro présente clairement une thématique: l'enfant, le fantastique, l'art, le ciel, l'animal en liberté, le sport, l'adolescence, demain, l'environnement. La reconstitution des contenus des numéros, qui ne présentent pas leurs sommaires, permet de se rendre immédiatement compte de la diversité des angles d'approche de la question envisagée. En guise d'exemple, le numéro d'octobre 1962, consacré au «Fantastique»:

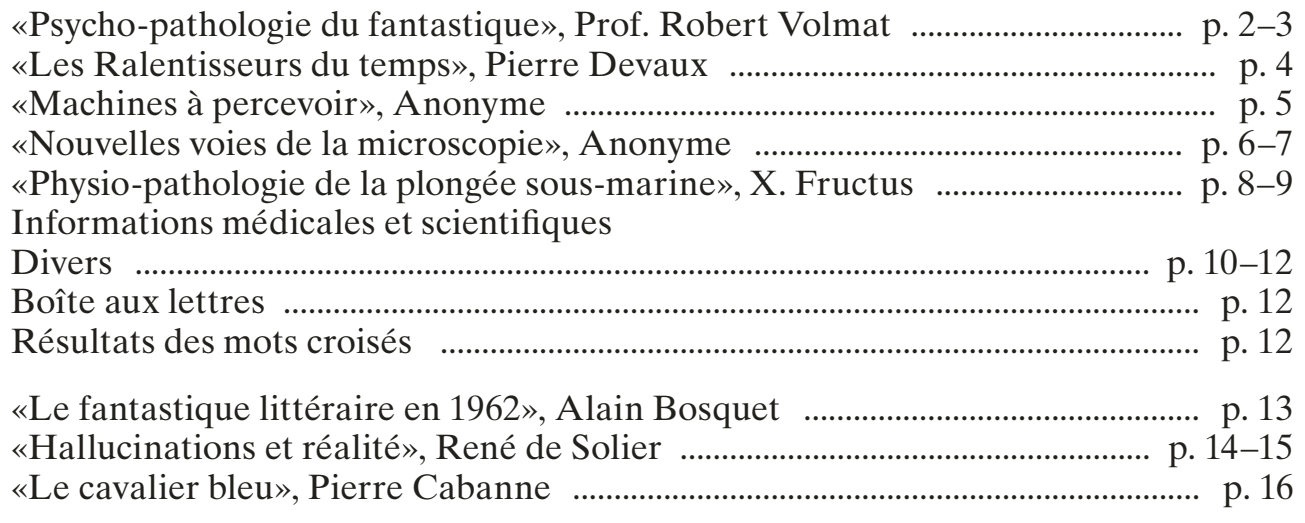




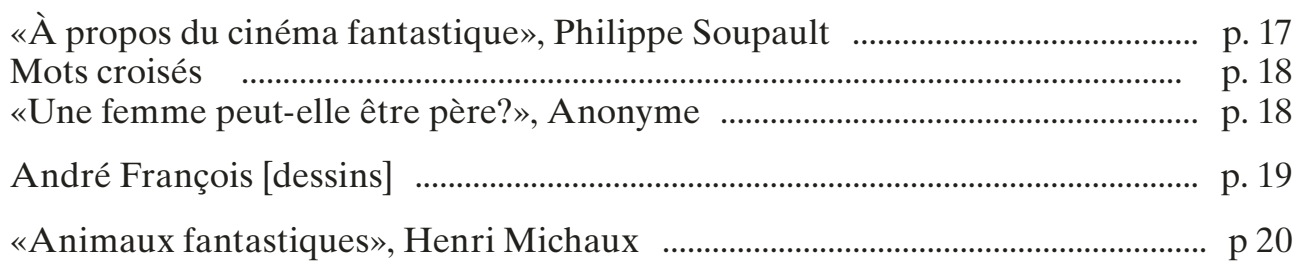

La thématique des livraisons n'empêche pas une grande diversité des questions abordées: l'art moderne, le cinéma, l'ergot de seigle, la notation musicale, les milieux socio-affectifs, les dermatoses, les mimiques dans les psychoses, la réanimation du nouveau-né, les techniques de survie, les appareils perceptifs, etc. Ce qui détermine le sujet est la plupart du temps son caractère expérimental, que ce soit dans les sciences ou dans les arts. À regarder de plus près le contenu de Sandorama, force est de constater que la revue a quelque chose de profondément non-rationnel, qui ne tient pas à la présence de la littérature et des arts, mais à son excentricité. Toutes les expérimentations scientifiques et artistiques de l'époque, de préférence les plus étranges, sont dans le journal.

Les textes littéraires parus dans Sandorama émanent d'auteurs reconnus et portent toujours, pour les auteurs vivants, sur le rapport entre médecine et arts. Le premier numéro présente ainsi des lettres, «Inédits de Max Jacob», présentées par Pierre-Michel Frenkel, et des «Notes sur la névrose et le théâtre» d'Eugène Ionesco. À ces textes de création et de critique littéraire s'ajoutent des contributions portant sur les arts plastiques, le cinéma dont l'importance pour la revue sera explicitée plus loin -, et la science. Dans l'ensemble, les articles se veulent avant tout informatifs, sont signés par des spécialistes, collaborateurs extérieurs auxquels sont passés des commandes, ou collaborateurs réguliers comme le neurobiologiste Henri Laborit ou l'historien de l'art Pierre Cabanne.

En revanche, l'énonciation du journal Sandorama est marquée par l'anonymat de la rédaction, fait caractéristique des parutions scientifiques de presse de la seconde moitié du XX ${ }^{\mathrm{e}}$ siècle, qui ne cherchent pas à ériger en figure humaniste un médecin littérateur, mais plutôt à garantir une objectivité incarnant l'institution. Un «nous» vient constamment alterner avec le logo des Laboratoires Sandoz pour prendre la parole dans les entretiens, s'occuper des rubriques généralistes, répondre aux lecteurs qui, dès le premier numéro, écrivent à la rédaction, dont l'adresse est à Bâle. On découvre finalement l'identité du rédacteur en chef dans le numéro du printemps 1964, grâce au courrier aux lecteurs annonçant que Michel Breitman a reçu un prix littéraire pour son roman Sébastien. Écrivain et traducteur (en particulier de Dino Buzzatti), Breitman est engagé au service de la propagande de Sandoz 
depuis 1961, où il dirige Sandorama en homme de lettres, c'est-à-dire en montrant une conscience aiguë des formes littéraires, de l'énonciation et de la figure du lecteur. Les médecins, auxquels est destinée la revue, se montrent très réactifs aux articles, soucieux de contester ou corriger une information ou demander des précisions supplémentaires. La réponse de la rédaction est alors souvent une adresse à un «cher lecteur». Dans le cadre de la revue, le médecin est donc avant tout un homme qui lit, un homme du commun, au même titre que son patient, pour lequel il doit néanmoins choisir les médicaments dont on lui fait la réclame. L'apparition de cette figure du lecteur dans l'histoire médicale des rapports entre le médecin et le patient mériterait à elle seule une longue analyse. Pour l'heure, il s'agit de saisir les questions que cela pose en termes de stratégie d'énonciation à partir du cas Sandorama.

À travers la figure du lecteur, le système énonciatif de la revue tisse une relation fiduciaire, relation économique de confiance entre un individu potentiellement client et un «nous» anonyme, qui l'englobe dans la communauté Sandorama ainsi formée. Au sein de cette dernière règne une manière de complicité dans les échanges, la revue s'appliquant à diversifier les modalités phatiques de ses énoncés. Sandorama lance ainsi des appels aux lecteurs pour qu'ils envoient, par exemple, des photographies de monuments saccagés ou détournés de leur fonction, manière de saisir des bizarreries architecturales, de se placer dans une politique de protection des œuvres d'art et du patrimoine, mais aussi de produire du lien et de la reconnaissance: «Nous continuerons dans les numéros à venir de Sandorama de publier les meilleures photographies qui nous seront envoyées.» Le journal implique donc le médecin-lecteur dans sa rédaction, un acte de partage qui produit une communauté. Les membres de cette dernière connaissent aussi la la solution des mots croisés: le nom de l'un des derniers médicaments de la firme.

Dans Sandorama, l'humour est de mise, dont on sait qu'il se fonde sur une prise de distance du locuteur vis-à-vis de l'énoncé. ${ }^{14} \mathrm{Si}$ le journal cherche à informer les médecins du progrès scientifique (les découvertes en microscopie, par exemple), de nouveautés en art (les expositions de Jean Arp et de Pablo Picasso), et n'hésite pas non plus à aborder des questions éthiques (les animaux de laboratoires), c'est dans un contexte général qui pose sur le monde un regard distancié, et un humour quelque peu désabusé. L'humour en appelle à différentes procédures énonciatives et visuelles ludiques, et nous n'en mentionnons ici que quelques-unes. Au sein des rubriques rédigées par la rédaction de manière anonyme, on constate le recours fréquent à ce que le philo-

14 Ducrot 1984; Genette 2002.

Gesnerus 77 (2020) 
logue André Jolles a bien nommé les «formes simples», ${ }_{15}^{15}$ une littérature populaire où la figure de l'auteur disparaît au profit de la collectivité qui en véhicule de bouche à oreille les contenus: le jeu de mots, la devinette, le conte. Le premier numéro s'ouvre ainsi sur un «Conte édifiant», l'histoire de trois naufragés auxquels est posée la question de savoir comment reconnaître un énoncé vrai d'un énoncé faux. Au lecteur qui ne pourrait pas répondre (on sait que seul le second naufragé trouve la réponse, mais pas quelle est la réponse), il est recommandé de chercher cette réponse dans le numéro. Le journal soigne donc d'emblée sa rhétorique, la captatio benevolentiae cherchant à s'attirer la bienveillance du lecteur en l'impliquant dans un jeu de piste. De la même manière, le journal recourt à des formes visuelles populaires: en témoigne la série des «Rébus médical en 1837», qui fait référence à «l'humour de nos collègues d'antan», dont on trouve des traces dans la poésie des carabins des revues médicales du XIX ${ }^{\mathrm{e}}$ siècle. ${ }^{16}$ (fig. 6). Il faut noter ici le vide bibliographique sur la question de l'humour en médecine, alors même que Sandorama affirme s'inscrire dans une tradition. On se souviendra que la question de l'humour médical relève aussi de l'ancienne tradition de la thérapeutique par le rire. Cette dernière est marquée par les pseudo-lettres d'Hippocrate aux Abéritains sur la maladie du philosophe Démocrite qui rit de tout et de rien. ${ }^{17}$ De surcroît, le sens commun sait que le sacerdoce médical est ponctué

\section{UN RÉBUS MÉDICAL EN 1837}
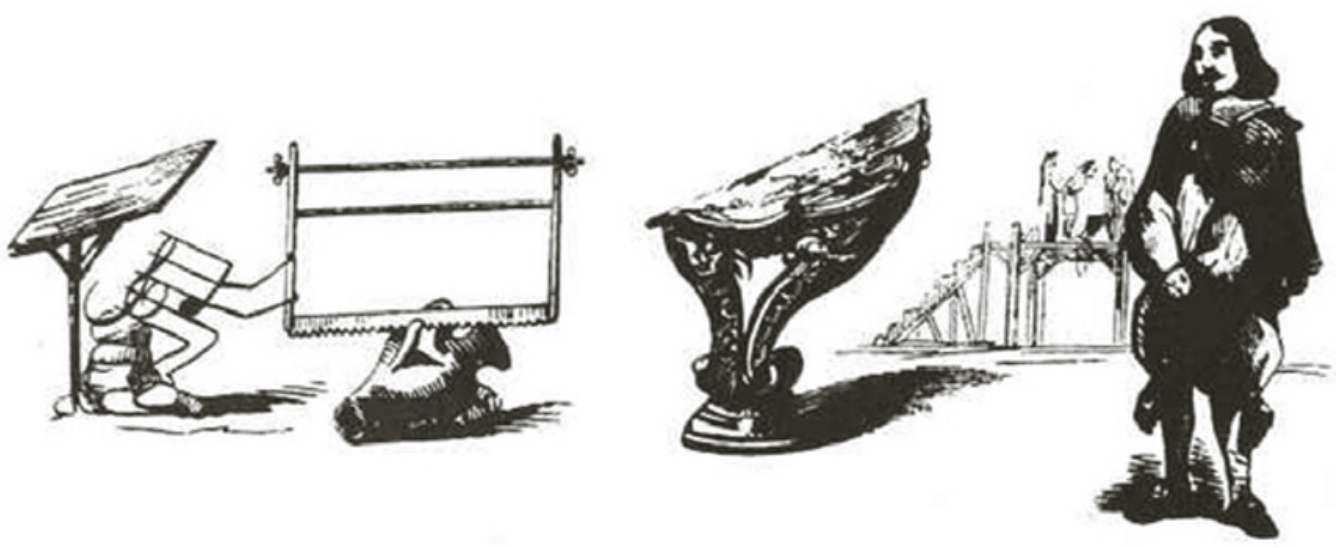

Fig. 6. Rébus paru dans Sandorama (automne 1963). Solution: La scie anse console de Thou (La science console de tout).

15 Jolles 1930.

16 Marchal 2018; Aron 2018.

17 Pigeaud 1981. 
de moments de détente permettant de tenir face à la maladie et à la mort. Mais jamais cette question n'a été véritablement problématisée et pensée, ce qui constitue pourtant un sujet à part entière pour les humanités médicales. Toujours est-il que cette mise à distance des émotions par l'humour peut prendre l'anatomie et les organes comme matériaux: c'est l'objectif du «courrier du cœur» de Sandorama, qui n'a rien à voir avec la rubrique des rencontres amoureuses, puisque c'est un exposé régulier des nouveautés scientifiques et pharmaceutiques en cardiologie, domaine où Sandoz excelle, l'organe étant de surcroît un élément graphique récurrent dans les publicités du journal (fig. 7). À plus d'un titre, donc, Sandorama s'impose comme un journal qui a de l'humour et du cœur, ne cherchant pas dans les canulars à mettre le lecteur mal à l'aise, mais à partager avec lui une mise à distance critique visà-vis de la maladie et de la science. L'ancienne division entre cardio-centrisme et encéphalo-centrisme, ${ }^{18}$ émotion et raison, est ainsi repensée par la voie des formes énonciatives et littéraires.

Invité sinon à rire, du moins à sourire, le lectorat de la revue trouvera dans ses pages de quoi prendre un peu de recul vis-à-vis de son métier, et se divertir. La rubrique «Informations médicales et culturelles» est sur ce point parlante, qui fait état de toutes sortes d'excentricités perceptives au fil des numéros: «entendre avec ses dents», «voir avec ses mains»; ou encore, elle entretient ses lecteurs de «nourritures célestes»: «Le Docteur Sydney Schwartz vient d'annoncer que certaines parties des engins envoyés sur la lune seront comestibles! Elles seront en effet constituées de lait en poudre, de maïs, de bananes, de diverses protéines.» Sandorama ne cesse d'en appeler au sens de l'humour du lecteur et au bon sens du médecin pour faire la part des choses, supposant son public intelligent, cultivé, et de la sorte, particulièrement valorisé.

À un tel public, la revue doit donner des articles de qualité en suivant un principe littéraire et artistique que Sandorama met un point d'honneur à respecter: le caractère inédit des textes, dessins, publicités que publie la revue. Les Laboratoires Sandoz affichent en même temps de manière implicite les moyens financiers qu'ils donnent au journal pour le plaisir du lecteur, le coût de l'inédit exigeant que les contributeurs soient largement payés. ${ }^{19}$ Sempé, Ylipe, Bosc, Chaval donnent ainsi à chaque numéro une nouvelle illustration. Dans le courrier des lecteurs, la rédaction répond à une demande à propos d'un texte de Roger Vadim: «Mais, cher lecteur, c'est l'article intégral que vous avez lu dans Sandorama, et il était parfaitement inédit, comme le sont

18 Barras 2019.

19 Les archives Sandoz ne renseignent pas sur ce point, les chiffres exacts apparaissant peutêtre sur les contrats des prestataires. 


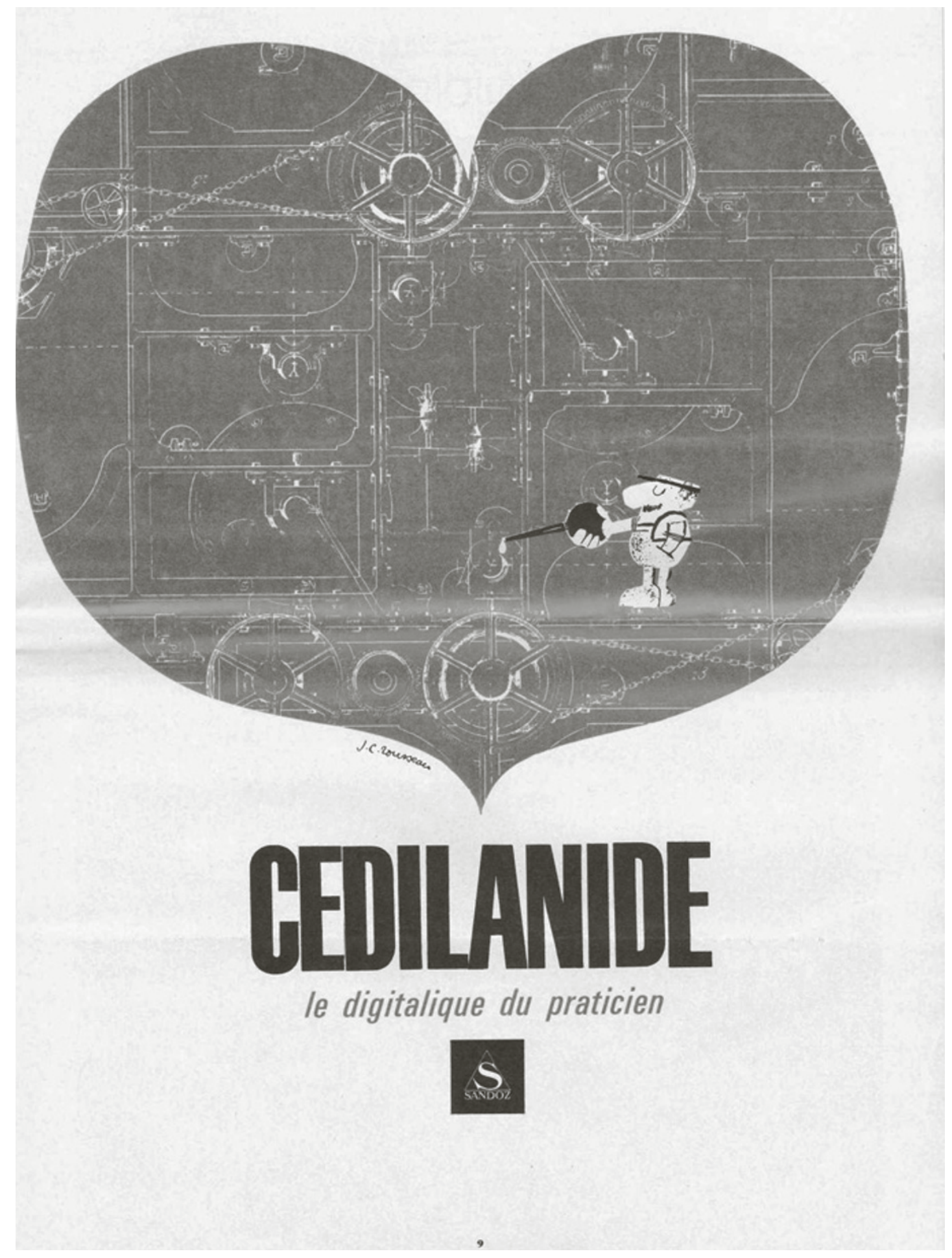

Fig. 7. Publicité dans Sandorama (1964).

82 Gesnerus $77(2020)$ 
toujours tous les textes de notre revue.» Le premier numéro de Sandorama présentait déjà des «inédits de Max Jacob» en reproduisant les archives manuscrites en question, fragments de lettres du poète disparu et plusieurs dessins. L'inédit donne ainsi depuis le début son label de qualité à la revue et reflète aussi la qualité des médicaments dont elle fait la propagande publicitaire.

Dans les années 1960, le développement des médicaments psychotropes pour apaiser les troubles psychiques (Melleril, Calcibronat, Largactil) est une révolution médicale qui emporte les enthousiasmes ou soulève les scepticismes. La question de la confiance accordée aux laboratoires pharmaceutiques est alors centrale. Malgré l'incertitude des effets de certains médicaments, par exemple la Chlorpromazine, la camisole chimique tend à remplacer la camisole de force dès le début des années 1950. Pour certains psychiatres, c'est un moyen d'éviter la violence punitive dans les asiles où, conjointement, des méthodes de soin complémentaires à la médication sont développées qui recourent à la parole et la pratique artistique. Un article anonyme de Sandorama (implicitement attribué à la rédaction) porte sur l'asile de Saint-Auban et la pratique de l'ergothérapie dans le numéro de janvier 1963. Il y est question de la rédaction d'un journal par les pensionnaires, activité occupant une place importante dans le processus thérapeutique. L'illustration de l'article présente une photographie d'un homme de dos, un dessin d'aliéné à la main. La légende indique: «Le rédacteur en chef en train de choisir la prochaine couverture». L'effet de mise en abyme avec Sandorama est évident, nouvelle touche d'humour allant jusqu'à supposer que la revue est elle aussi un objet thérapeutique, les rédacteurs et les lecteurs devenant des aliénés dans une inversion carnavalesque des rôles entre médecin et patient.

L'attention aux formes dans Sandorama est manifestement redevable d'une culture de la Gestalt qui s'impose dans les espaces thérapeutiques depuis l'Allemagne dès l'Entre-deux-guerres. La parution de l'ouvrage de Hans Prinzhorn en 1922, Expressions de la folie (Bildnerei der Geisteskranken. Ein Beitrag zur Psychologie und Psychopathologie), fait référence en matière de Gestalt-thérapie, qui applique à la psychiatrie la lecture de l'expressivité formelle de manière décisive. ${ }^{20} \mathrm{C}$ 'est par les formes que s'exprime la folie et c'est dans les formes (dessins, peintures et écrits d'aliénés) qu'il est possible de la lire, assertion type que l'on retrouve encore chez Jean Delay. Ce dernier dirigera d'ailleurs une revue des Laboratoires Sandoz dont le titre, Psychopathologie de l'expression, fait référence à Prinzhorn, le psychiatre n'ignorant pas que l'ouvrage est tout aussi connu de ses collègues que des écrivains et des artistes dans son lien depuis 1945 avec l'Art brut, Paul

20 Starobinski 1984.

Gesnerus 77 (2020) 
Budry l'offrant d'ailleurs à Jean Dubuffet dans les années $1950 .{ }^{21}$ Certains philosophes et médecins estiment donc que pour comprendre la situation psychique d'un individu, l'introspection ne donne presque rien: mieux vaut lui substituer l'extériorisation de formes, dessins ou textes. À la notion de Gestalt est attachée l'idée que «ce qui est au-dedans est au dehors», ${ }^{22}$ les sentiments, les émotions, les sensations sont lisibles parce que visibles dans les comportements, les gestes, l'expression. L'heure est à la zoologie des formes animales ${ }^{23}$ au "gestalt switch», ${ }^{24}$ au biomorphisme artistique exemplifié par Jean Arp. ${ }^{25}$ Les revues pharmaceutiques suisses, et parmi elles Sandorama, participent de cette expansion du domaine des formes, en offrant à l'art graphique des possibilités de développement dont l'inventivité sans précédent traduit l'importance à cette époque d'une pensée par et des formes.

L'extraordinaire fécondité du graphisme pharmaceutique suisse des années 1960 est assurée par les collaborations des firmes avec le Verband schweizerischer Graphiker et la Allgemeine Gewerbeschule Basel dirigée par Armin Hofmann et Emil Ruder. Ce qui se joue dans ces années est la naissance d'un «Swiss style» que les historiens du graphisme nomment aussi le «Geigy style» ${ }^{26}$ tant la publicité réalisée pour les Laboratoires dans les domaines de la pharmaceutique et de la chimie agro-alimentaires a été propice à son développement en raison des moyens importants mis au service de la propagande. Si le nom de Geigy l'emporte, c'est parce que le laboratoire chapeaute en réalité les autres firmes. Dès la fin de la Seconde Guerre mondiale en effet, la plupart des industries chimiques suisses se regroupent pour la communication d'une image de marque nationale commune, et cela avant la fusion officielle des groupes au sein de Novartis (Ciba et Geigy en 1970, puis Sandoz en 1997). ${ }^{27}$

La publicité pharmaceutique en Suisse est soumise à un contrôle cantonal, voire supra-cantonal avant même la formation en 1971 de l'OICM (Office intercantonal de contrôle des médicaments). Des règlements concordataires existaient depuis 1900 pour l'examen et l'enregistrement par un centre commun des spécialités pharmaceutiques et des dispositifs médicaux. ${ }^{28} \mathrm{C}$ 'est à ces règles qu'obéissent les industries chimiques suisses se regroupant au début des années 1950 pour produire une campagne de publicité internationale promouvant une production locale et diffusion internationale, modernité des structures et des

21 Pic 2014a.

22 Rosenthal/Visetti 1999.

23 Portmann 1948.

24 Kuhn 1970.

25 Maldonaldo 2006.

26 Jansers 2009, 10.

27 Dettwiler 2011.

28 Campanelli 2018. 
états d'esprits. Cette campagne fait des choix graphiques résolument modernes et la publicité pharmaceutique écrit durant ces années l'une des pages les plus importantes de l'histoire du graphisme en Suisse. Geigy, dont le «Département de la propagande» (rebaptisé en 1966 «Département de la publicité») ${ }^{29}$ est dirigé par René Rudin jusqu'en 1970, recrute ses graphistes en Suisse mais aussi à l'étranger notamment Japon et États-Unis ${ }^{30}$. La firme impose une police publicitaire avec des principes stricts: une position objective; des éléments suggestifs; une excellence du niveau artistique, de la qualité des illustrations, du design typographique et de la réalisation technique; la volonté de créer un «Geigy style» et, enfin: «All printed material that leaves our house and every other statement of our propaganda should express the trustworthiness of the name Geigy, and thus also function as goodwill propaganda in addition to the immediate objective». ${ }^{31}$ Fiabilité du nom et bonnes intentions de la propagande, qualité artistique et réalisations impeccables, tels sont les principes de la publicité pharmaceutique des années 1960 qui fait coïncider «Swiss style» et «Geigy style».

Le volume trilingue Publicité et Graphisme dans l'industrie chimique de 1967 analyse ce mouvement de l'avant-garde graphique dont il est contemporain. Largement illustré, réalisé par Hans Neuburg, avec la participation de René Rudin, Armin Hofmann et Emil Ruder, mais aussi Victor Cohen, le volume réunit quelques-unes des personnalités les plus importantes et les plus actives de l'époque dans le domaine. Il propose une typologie des graphismes publicitaires dans l'industrie chimique, l'agrochimie, la pharmaceutique. Pour ce domaine, le volume précise que les réalisations graphiques varient selon qu'elles s'adressent aux médecins ou aux malades, dernier cas concernant les médicaments en vente libre. Un autre facteur conditionne les formes publicitaires: leurs supports. Il peut s'agir d'une affiche destinée à être placardée dans la rue ou de prospectus et bulletins publicitaires pour la marque, envoyés directement aux médecins. Dans tous les cas, la publicité doit opter pour des solutions graphiques qui, comme pour les emballages des médicaments, sont à la fois sobres et inventives: «une synthèse d'information pure, de publicité vivante, et d'un graphisme équilibré». ${ }^{32}$ Le Bulletin Sandoz est donné comme exemple de publicité de prestige, car il fait appel à des artistes ou utilise des dessins d'enfants, le laboratoire se distinguant par son attention continue à l'endroit de la création et ses collaborations avec des créateurs. La typographie des graphismes utilisés est la suivante: «jeux de caractères», «surfaces colorées», «diagrammes et courbes», «poumons et formes des pilules», «nature», «coupes histologiques»,

29 Junod 2009. 32

30 Jansers 2009, 20.

31 Junod 2009, 32

32 Neuburg 1967, 27.

Gesnerus 77 (2020) 


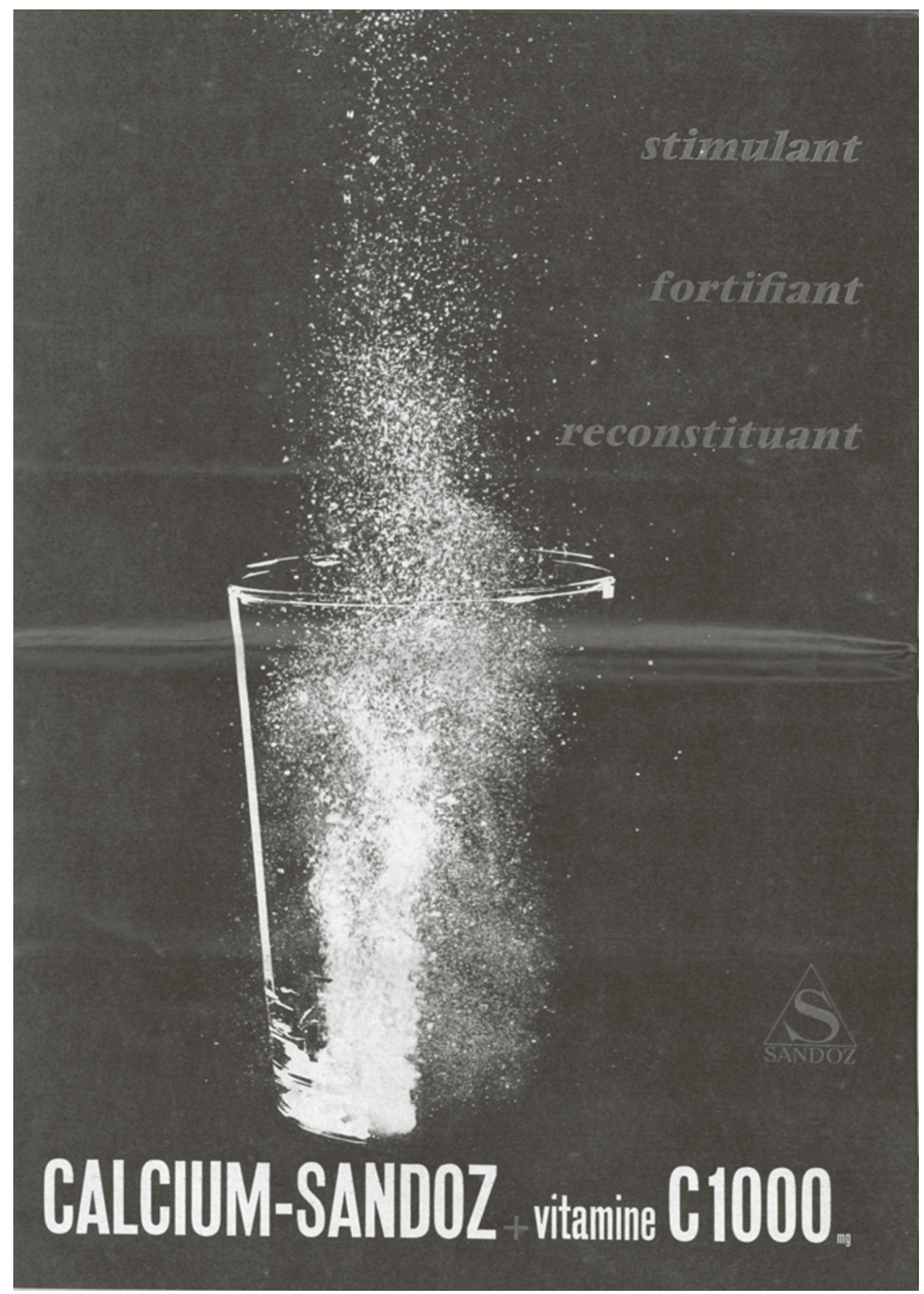

Fig. 8. Publicité dans Sandorama (1964). 


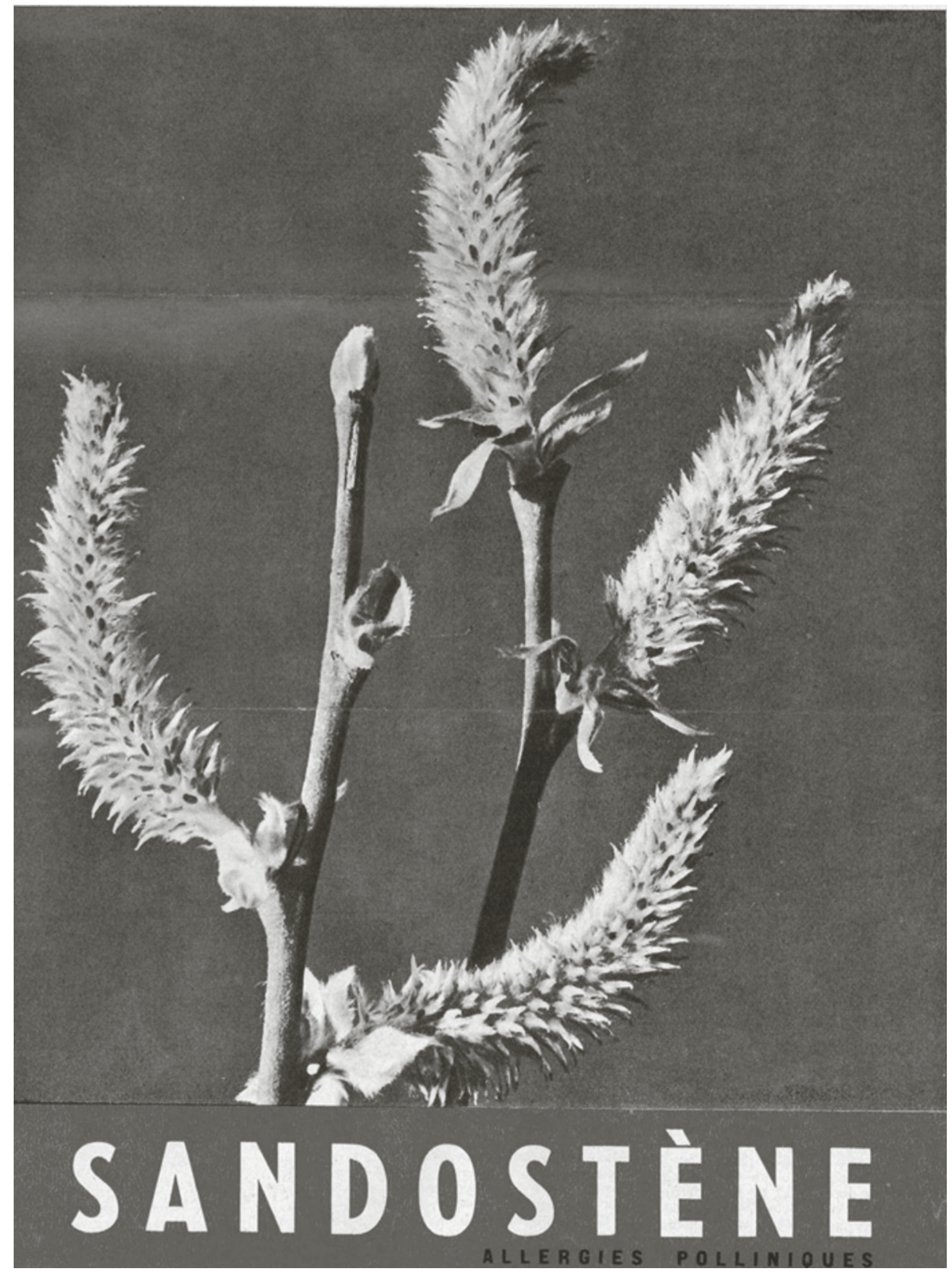

Fig. 9. Publicité dans Sandorama (1962). 


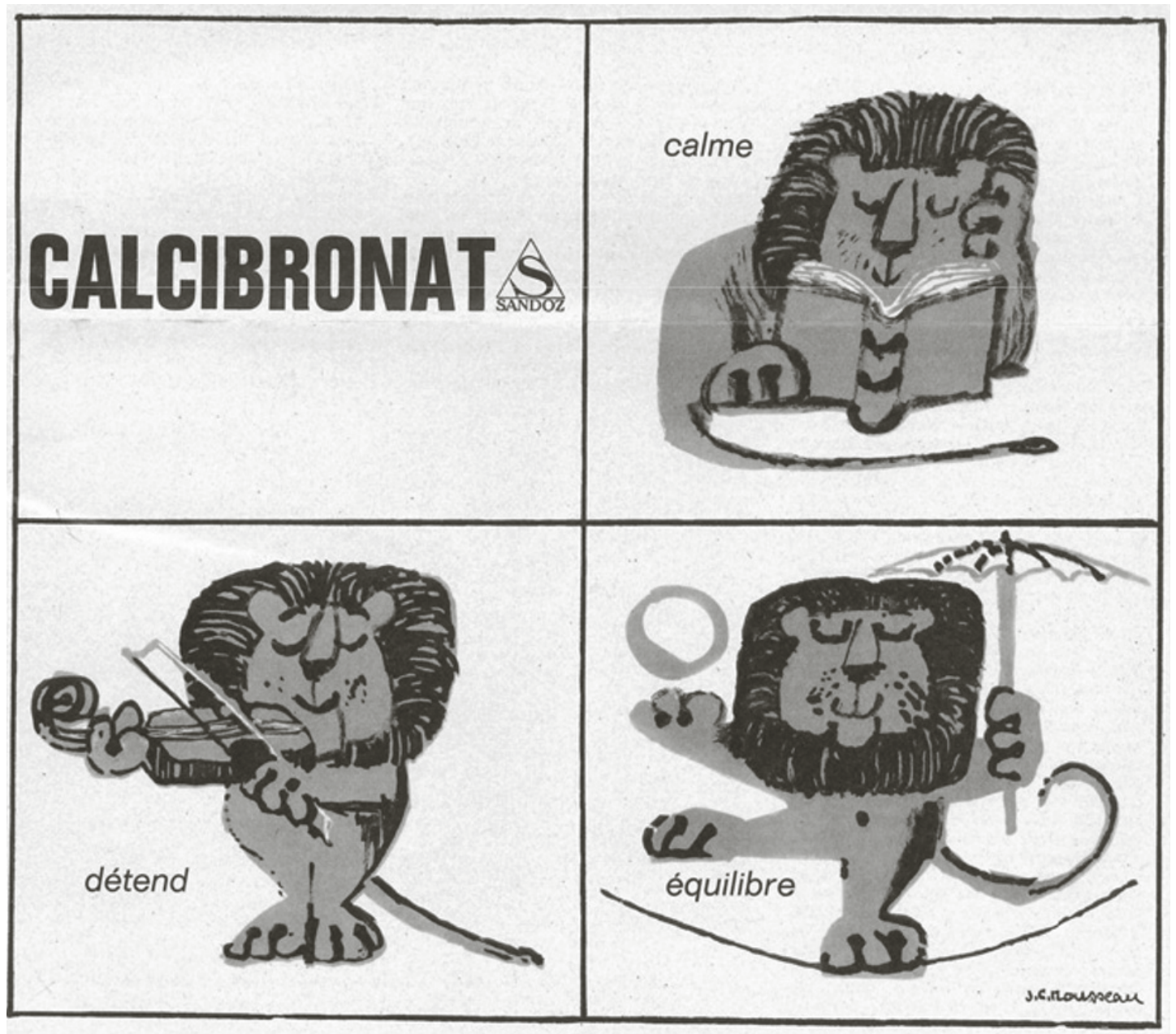

Fig. 10. Publicité dans Sandorama (automne 1963).

«éléments abstraits», «dessins et stylisation», «les flammes», «les articulations», «photo-graphisme», «photographie», «zoologie», «photomontage», «emploi systématique d'un motif», «gouttes d'eau», «fibre musculaire», ${ }^{33}$ etc.

Autant de principes graphiques que l'on retrouve dans Sandorama (fig. 8 et 9), la revue étant aussi le lieu d'une importante expérimentation des formes visuelles publicitaires. Chaque numéro renouvelle le graphisme publicitaire en fonction du thème (fig. 10, pour le thème de l'animal). Elles participent d'une communication qui mise sur l'esthétique pour produire le plaisir et la détente, susciter la complicité et la confiance. Si Sandorama n'échappe pas à ses principes élargis à toute la publicité pharmaceutique de l'époque, malgré la concurrence et la compétition entre les firmes, elle possède aussi sa propre identité graphique (noir/blanc/rouge), l'unité dans la variété étant un impératif de la propagande de marque.

33 Neuburg 1967, 27. 


\section{L'expérimentation des formes cinématographiques: le montage dans Sandorama}

Dans l'abondante iconographie de Sandorama, le film scientifique et le cinéma de la Nouvelle vague occupent une place déterminante, la physionomie de la revue pouvant se résumer en un montage entre une revue littéraire d'avant-garde, le modernisme graphique du «Swiss style» et l'avant-garde des Cahiers du cinéma. Les Laboratoires Sandoz ont en effet développé dans les années Soixante ce que Thierry Lefebvre nomme «l'art subtil de la communication audiovisuelle médicale». ${ }^{34} \mathrm{Ce}$ dernier souligne également l'importance dans ce processus du rédacteur en chef de Sandorama qui, à partir de 1966, dirige la Cinémathèque Sandoz sise dans les locaux de la filiale, rue de Penthièvre (8e):

Ce grand cinéphile s'enthousiasme pour le projet d'une cinémathèque d'exception, dont l'un des axes de prédilection sera la psychiatrie. Il est bientôt rejoint par le jeune Jean-Charles Gaspard, lui-même passionné par le septième art et condisciple en cinéphilie de Bertrand Tavernier. Dotés d'un enthousiasme communicatif et de budgets généreux, les deux hommes n'hésitent pas à produire des films médicaux d' "art et d'essai» parfois très ambitieux, comme, par exemple, Images d'un monde visionnaire d'Éric Duvivier (1963), qui tente de reproduire au cinéma les hallucinations sous mescaline et haschich consignées dans ses ouvrages par Henri Michaux, ou L'Ordre de Jean-Daniel Pollet (1973), qui dénonce le sort indigne pendant longtemps réservé aux lupiques. Cet «âge d'or» du film médical prend fin au début des années 1980, avec la montée en puissance du marketing et de la publicité, qui finissent par absorber les services de relations publiques. Les chefs de produit imposent désormais des stratégies commerciales visant la rentabilité à court terme. ${ }^{35}$

Sous l'impulsion de Breitman, la revue Sandorama est donc intimement liée aux activités cinématographiques des laboratoires Sandoz qui complètent les activités éditoriales. ${ }^{36}$ Le recours au support cinématographique a une triple fonction: d'une part, les films restituent des expérimentations et restent à usage interne. Ils sont alors d'une facture extrêmement simple, sans générique, ni scénario. D'autre part, le cinéma est utilisé à des fins pédagogiques pour diffuser les connaissances scientifiques sur les nouveaux médicaments. Enfin, ils sont réalisés pour la propagande, comme par exemple la série de reportages intitulés Sandorama, filmés sur les sites des laboratoires Sandoz à Bâle et à l'étranger en 1960. La revue, quant à elle, se montre attentive au développement du cinéma médical en lui donnant une place au sein du ci-

34 Lefebvre 2014, 393.

35 Lefebvre 2011, 141.

36 Sandoz, qui produit le film de Henri Michaux et Eric Duvivier a également commandé au psychiatre Julian de Ajuriaguerra un ouvrage sur Michaux qu'il cosignera avec François Jaeggi en 1959, Le poète Henri Michaux et les drogues hallucinogènes. Contribution à la connaissance des psychoses toxiques. De son côté, Geigy publie dans la série des Acta psychomatica la thèse de médecine de Jean Starobinski en 1960, Histoire du traitement de la mélancolie des origines à 1900. Pic 2014b et 2016a, Leibovici 2014. 
néma d'art et d'essai, ce qui sera ensuite le fer de lance de la revue Médecine/ Cinéma (1968-1973) émanant de la Cinémathèque Sandoz. ${ }^{37}$

La maquette de Sandorama évoque par ailleurs celle des Cahiers $\mathrm{du} \mathrm{ci-}$ néma, malgré la différence de format d'avec le cahier d'écolier de la revue de la Nouvelle vague, dirigée jusqu'en 1963 par Eric Rohmer puis Jacques Rivette. Les Cahiers se présentent dans les années 1960 avec une charte graphique qui lie le jaune avec le noir et blanc, là où Sandorama travaille avec le rouge. On peut donner d'autres points de ressemblances avec la revue mensuelle, qui se veut anticonformiste et provocatrice, et qui défend le cinéma d'auteur contre le cinéma grand public: la mise en page se veut simple, sobre, dense, sans couleur, les images documentent davantage qu'elles n'illustrent, mais chaque photographie cherche un effet visuel et les légendes sont en italique. Les colonnes des articles ne marquent pas les intertitres de manière très appuyée. Le rapport du texte et de l'image se veut globalement réduit au plus simple, les mots cédant à l'image la responsabilité de provoquer un étonnement critique et une joie esthétique.

Dans les pages de Sandorama, on trouve également des images en référence directe avec la Nouvelle vague, notamment une photographie de JeanPaul Belmondo pour illustrer un article de J.-M. Aubry de mars 1965 sur «Les milieux socio-affectifs», l'image de l'acteur culte du mouvement (fig. 11) avec sa fille Patricia légendée comme suit: «Même en famille, le Don Juan ne perd rien de son prestige.» Mais l'ensemble de l'iconographie de Sandorama se veut surtout «photogénique» au sens que donnent à ce terme les cinéastes depuis Jean Epstein, c'est-à-dire capable de saisir l'invisible du réel, ce qui échappe à l'œil nu et surprend par sa beauté inattendue, réaliste et étrange à la fois. Le soin apporté au choix des images, photographies et photogrammes, dans les articles comme dans les publicités, témoigne d'une esthétique cinéphile résolument avant-gardiste. La publicité pour le Torécan (fig. 12), médicament contre les vertiges, suggère une scène de film à suspens, la peur et l'angoisse altérant la perception (est-ce le corps qui penche ou l'escalier?).

La référence au cinéma dans Sandorama s'élabore donc en regard de la politique des laboratoires qui produisent dans les années 1960 plus de deux cents films scientifiques ${ }^{38}$ diffusés dans les hôpitaux, les universités, lors de séances annuelles à la Cinémathèque Sandoz, mais aussi au Musée de l'homme, dont le ciné-club Art \& Science est un temps animé par Duvivier, en complicité avec Breitman. Ce dernier initie la réalisation de films scien-

37 Bonah/Danet 2018.

38 Lefebvre 2014. 


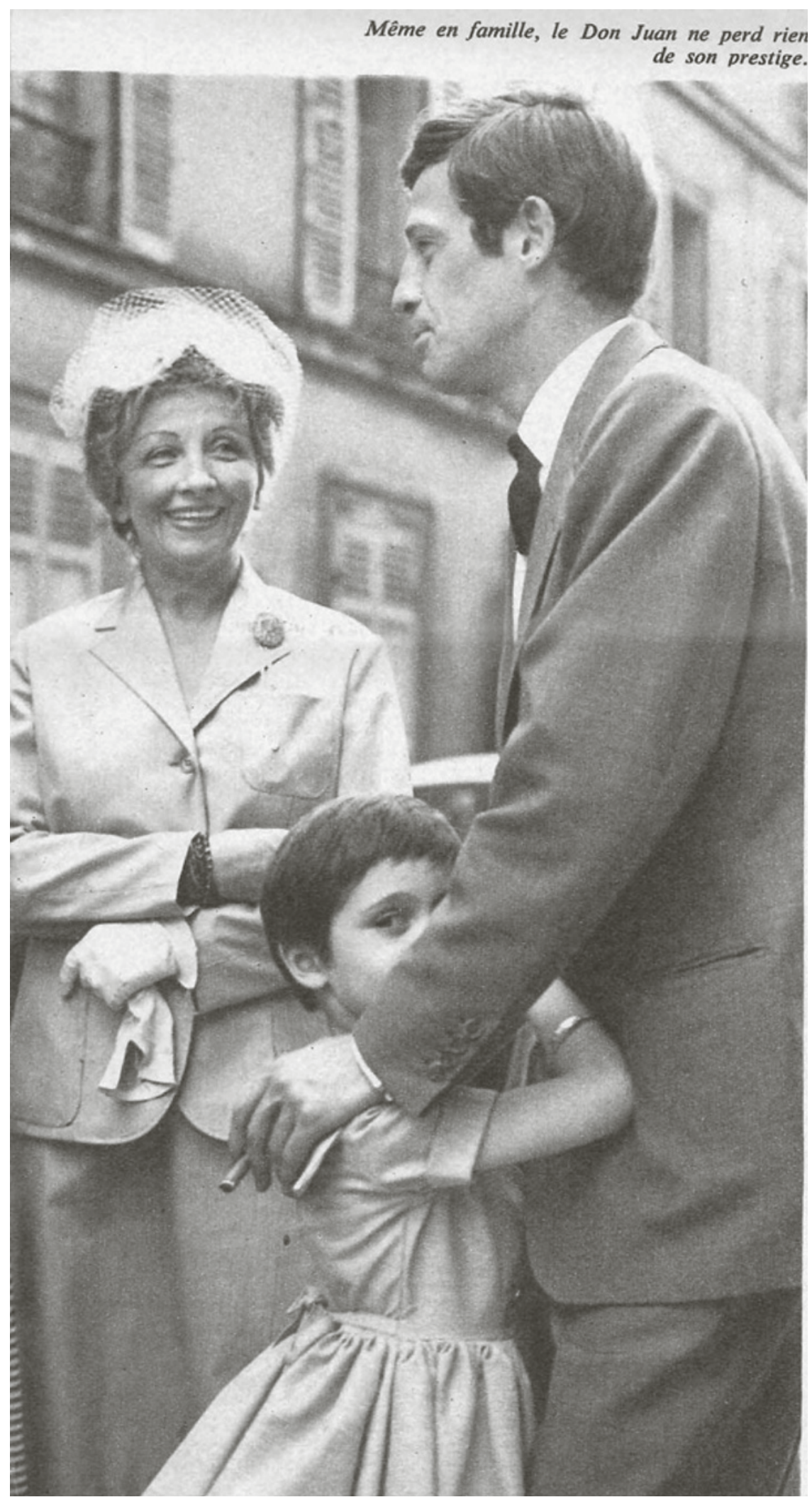

Fig. 11. Illustration de l'article «Les milieux socio-affectifs» par J.-M. Aubry, Sandorama (mars 1965). 


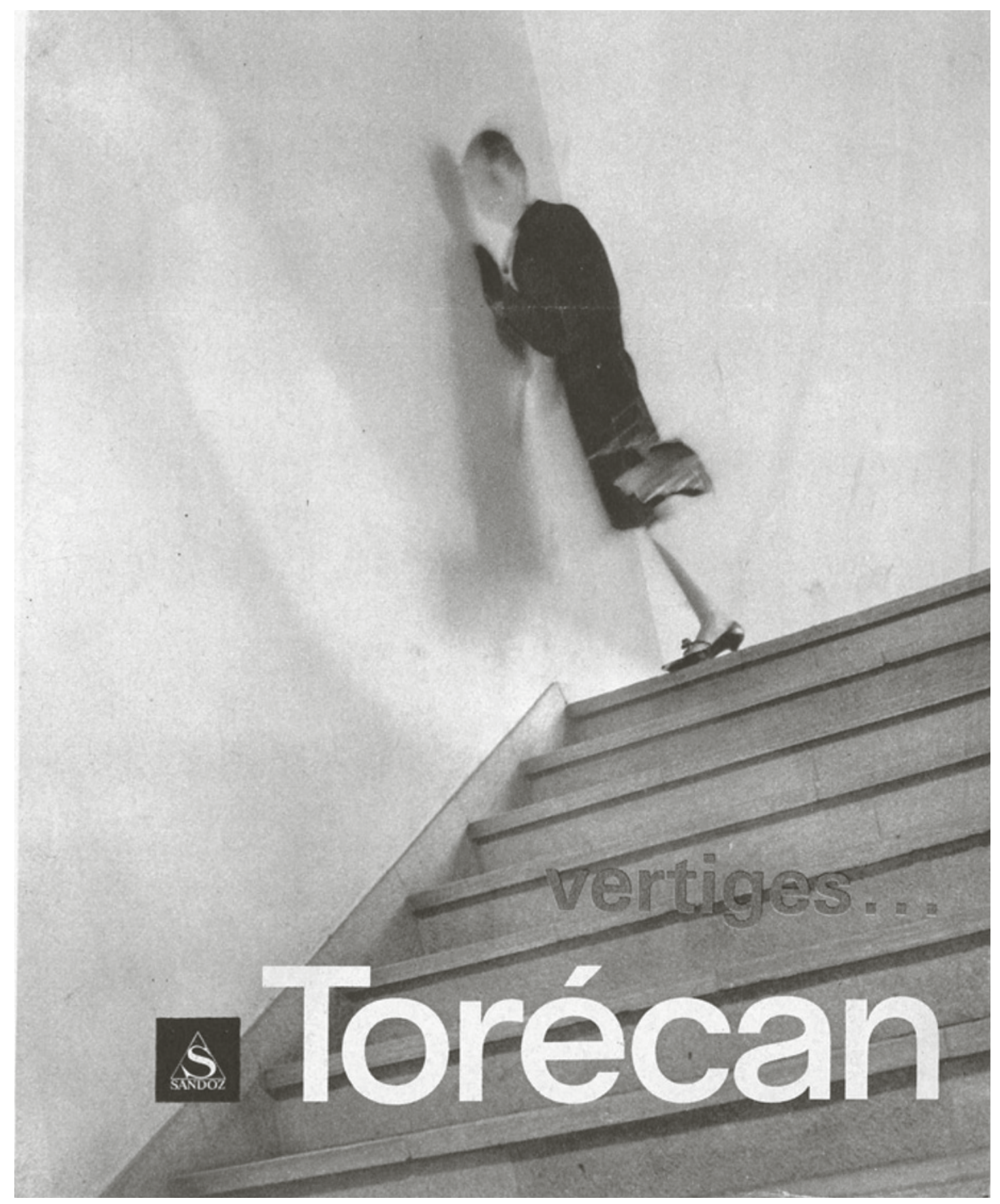

Fig. 12. Publicité dans Sandorama (1962).

tifiques d'avant-garde comme l'adaptation du livre de Gérard de Nerval, $A u$ rélia, par Anne d'Astrée en 1964. Sandorama publie à l'automne 1964 un encart sur «Les films Sandoz et la presse» qui donne un florilège de citations sur la réception de ce film et de celui de Michaux: «Bien que les séances de films que nous organisons soient réservées au Corps médical, il n'est pas rare que la grande presse en fasse état.» Ces productions scientifico-littéraires 


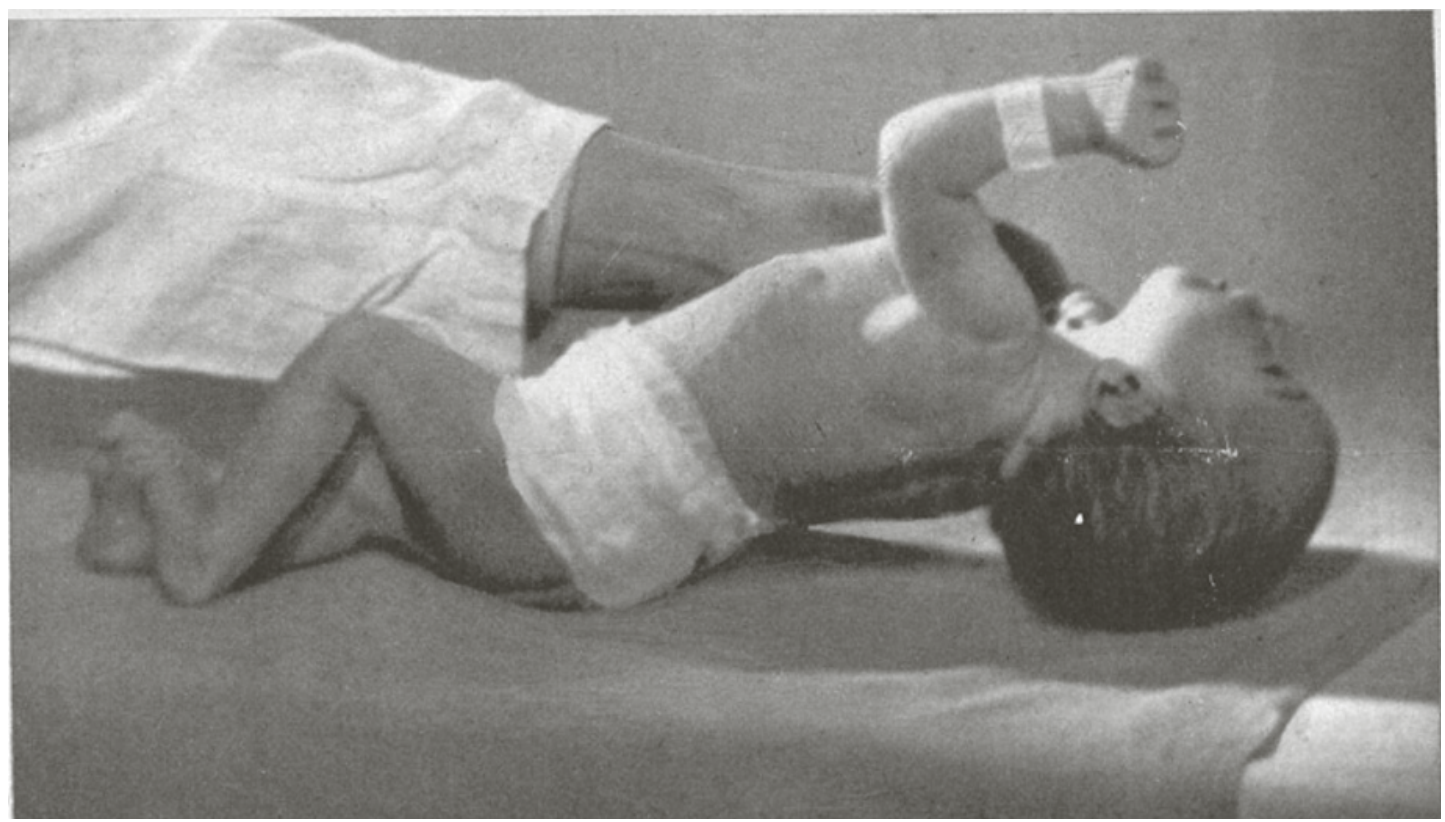

Fig. 13. Illustration de l'article «Détresse respiratoire», Sandorama (juillet 1962).

apportent un éclairage décisif sur la manière dont, par les formes, science et arts convergent autour d'un même thème touchant au versant irrationnel de l'humanité (le rêve et l'hallucination, par exemple). Sur ce point l'esprit de Sandorama est représentatif de celui d'une époque où la médecine cherche à investir par les arts des domaines fermés à une démarche strictement positiviste. De surcroît, le film est aussi un outil de travail interne pour les expérimentations scientifiques, dont des photogrammes illustrent les articles de Sandorama, par exemple sur la réanimation du nourrisson (fig. 13), moment suspendu entre la vie et la mort, image saisie par la caméra à des fins de démonstrations didactiques et cognitives. Car le film est avant tout un outil pédagogique pour communiquer un progrès scientifique, et on pense ici au film de Roger Heim sur la psilocybine ou sur l'écologie, Nature morte (1967). ${ }^{39}$ Les Laboratoires Sandoz financent ainsi un ensemble faramineux de films dans les années Soixante, qui rivalisent avec le cinéma expérimental, en particulier dans les génériques où l'on retrouve la qualité et l'inventivité graphique des publicités pour la firme. Les films, qui émanent également du service de la propagande, sont en définitive aussi une vitrine pour la marque.

Mais le cinéma n'est pas seulement présent dans Sandorama comme thématique, il donne aussi à la revue un modèle formel: le montage. Ce dernier est, au moins depuis l'Entre-deux-guerres, propre au genre du manifeste des

39 Pic 2018b. 
avant-gardes avec le collage et le photomontage. Dans Sandorama, journal apolitique s'il en est, le numéro d'avril 1963 donne un photomontage en guise d'illustration d'un article anonyme de la section générale «Informations scientifiques et culturelles», intitulé «Vivre sous l'eau», dont on se demande s'il est un canular: «Des chercheurs de l'université de Leiden, sous la conduite du docteur Johannes Kylstra ont tenté, avec succès, une très intéressante expérience: faire vivre et respirer des mammifères sous l'eau.» La description d'une augmentation de l'oxygène dans un bassin et la description des résultats (noyade des souris et survie des chiens par drainage des poumons) semble pourtant bien véridique. L'image du chien dans le bocal, photomontage de Serge Hambourg (fig. 14), photographe collaborant régulièrement à Sandorama, porte un regard critique sur la science qui tend à devenir science-fiction, sans pour autant discréditer l'expérience, dont la fin du texte nous rappelle qu'elle est liée aux tentatives de faire survivre un fœtus humain ex-vitro. Outre ce photomontage, force est de constater que la totalité de la maquette de la revue obéit à une logique de montage qui cherche à produire une expérience d'étonnement et de choc chez le lecteur, non sans lui faire esquisser un sourire.

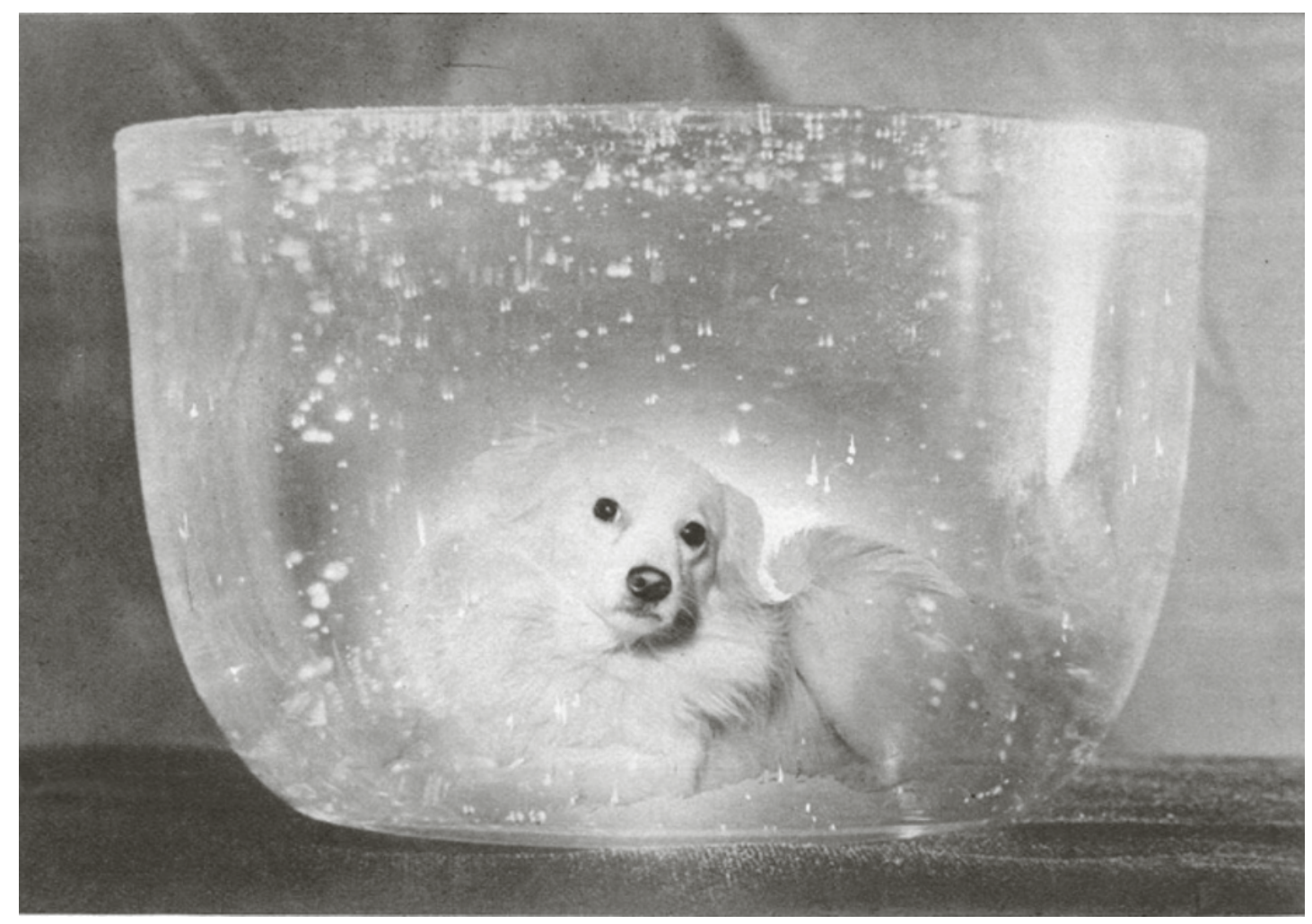

Fig. 14. Illustration de l'article «Vivre sous l'eau» par Anonyme, Sandorama (avril 1963). 
Sur ce point, le coup de force de Sandorama se trouve dans la dernière de couverture des numéros de la première série, qui présente toujours un texte littéraire inédit, qui est accompagné d'une publicité pour un médicament. Ce type de montage est présent jusqu'au printemps 1964, les deux derniers numéros au format tabloïd l'éliminant, déjà orientés vers une redéfinition de la ligne éditoriale. L'ensemble forme un corpus de dix textes traitant du rapport entre médecine et arts signés par Eugène Ionesco, Roger Vadim, Henri Bosco, Michel Butor, Serge Bourguignon, Henri Michaux, Alain Robbe-Grillet, Jean Paulhan, Dominique Aury. Par le montage entre un texte littéraire, par exemple, celui d'Alain Robbe-Grillet (fig. 15) sur l'expérience vertigineuse du temps dans le récit moderne, et un médicament, en l'occurrence psychotrope, le Calcibronat contre l'irritabilité, les sautes d'humeur et les difficultés du sommeil, la dernière de couverture laisse ouverte l'interprétation des rapports entre littérature et thérapeutique.

Prenons l'exemple de Robbe-Grillet, qui propose une réflexion sur le récit moderne d'un temps chronologique et ses ruptures vis-à-vis du récit classique: c'est une expérience vertigineuse où n'existe plus que la spirale infinie d'un présent sans repère d'hier ou de demain. Un pur ici et maintenant qui, pour Robbe-Grillet, est au centre du film d'Alain Resnais L'Année dernière à Marienbad de 1961, dont il a écrit le scénario et réalisé le découpage. Il commence par ces mots: «On a beaucoup répété que le temps était le personnage principal du roman contemporain.» Le temps dont il est question est un présent cénesthésique, où la perception surdéterminée du fait d'être là et d'exister produit un état de trouble vécu non pas seulement par les personnages, mais aussi par le spectateur d'un film. Car, selon les théories de la réception cinématographique, le cinéma se déroule avant tout dans la tête de ce dernier, dans ce qu'il produit d'images tierces grâce au montage. Le fait est acquis dès les premières théories du cinéma que l'on doit aux formalistes russes, ces derniers estimant que «le processus de discours intérieur du spectateur» était la véritable nouveauté du cinéma et résidait dans le montage: «Pour l'étude des lois du cinéma (avant tout du montage), il est très important de reconnaître que la perception et la compréhension du film sont indissolublement liées à la formation de ce discours intérieur qui relie entre eux les cadres isolés. Le discours intérieur du spectateur.» ${ }^{40}$ Le cinéma ne révolutionne pas seulement notre rapport au temps et à l'espace en inventant l'image-mouvement, il bouleverse aussi la perception et l'horizon de la réception de tout message, de là son importance politique comme support de propagande au XX ${ }^{\mathrm{e}}$ siècle. Dans Sandorama, le montage des images entre elles,

40 Eikhenbaum 1927, 45.

Gesnerus 77 (2020) 


\section{le temps dans le récit moderne}

par

\section{ALAIN ROBBE GRILLET}
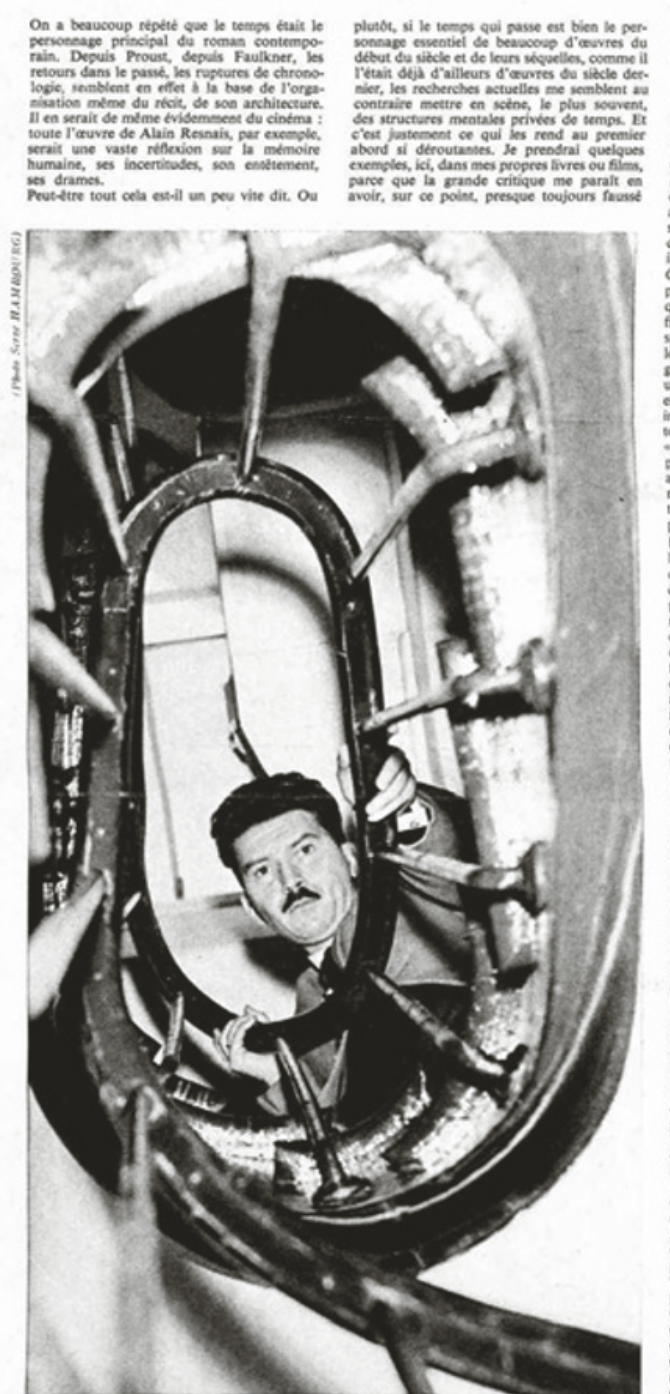

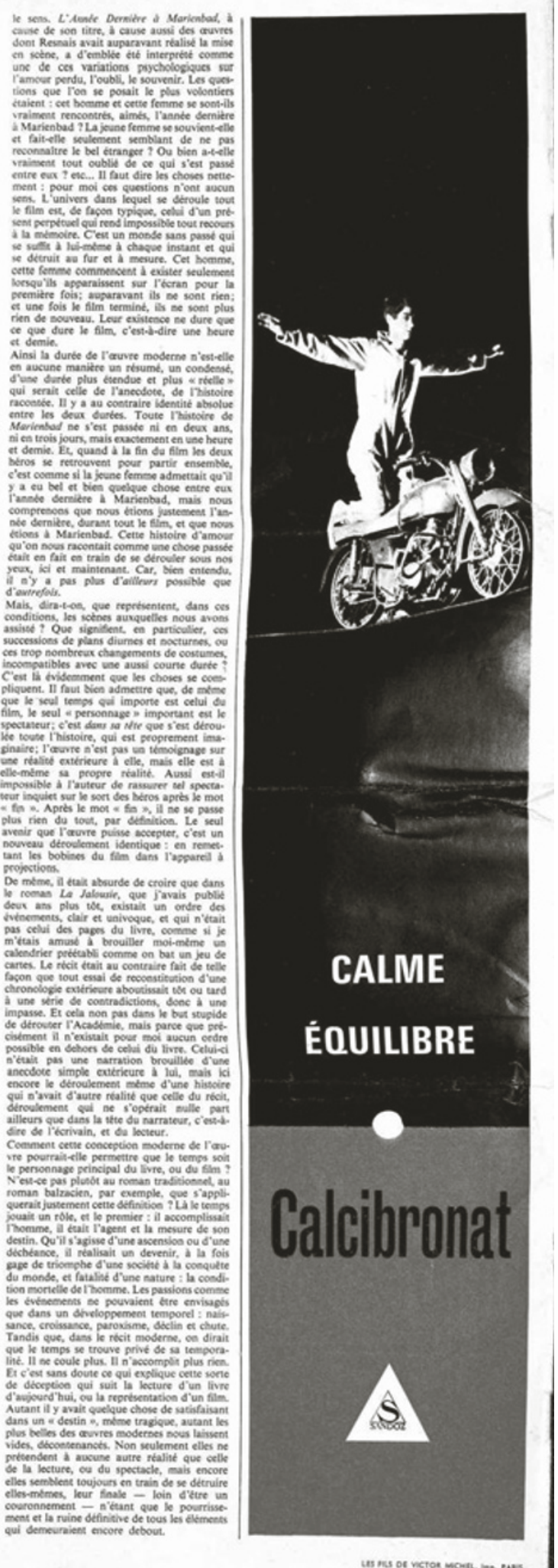

Fig. 15. Dernière de couverture du numéro de Sandorama (avril 1963). 
qui commande la réalisation de la maquette de la dernière de couverture, produit un étonnement, une inquiétude, selon une modalité propre au montage ${ }^{41}$, tel que le pratiquaient les revues de l'avant-garde artistique et littéraire de l'Entre-deux-guerres (par exemple la revue Documents de Georges Bataille et Michel Leiris). Outre les limites de la science face aux troubles de la perception, c'est la fonction thérapeutique des arts, dans leur rapport surdéterminé aux états modifiés de conscience, qui est politiquement interrogée. Rien comme le temps cénesthésique du récit moderne selon Robbe-Grillet n'est à même de susciter le vertige de la raison.

Le montage des images de la dernière de couverture, qui met en rapport l'infini du temps et de l'espace (la spirale de l'escalier et le funambule à la moto du cirque), mais aussi la littérature (le récit moderne) et le médicament psychotrope, suscite une interrogation critique chez le lecteur-spectateur-médecin: qu'est-ce donc que le pharmakon: la littérature ou le médicament? Et dans les deux cas, s'agit-il du poison ou du remède? ${ }^{42}$ Le chiasme reste impossible à résoudre, tandis que les questions peuvent s'enchaîner: et que peut la science face au trouble existentiel qui saisit l'homme devant le monde perçu? Si le montage peut être pensé ici comme une nouvelle forme d'humour éminemment critique, ${ }^{43}$ et l'on n'oublie pas que le rédacteur en chef de Sandorama n'est ni scientifique ni médecin, on peut aussi former l'hypothèse que le journal traduit l'esprit d'une époque: Merleau-Ponty a publié Signes en 1960, où sa critique du cartésianisme est un éloge de l'inquiétude scientifique et de l'étonnement philosophique. ${ }^{44}$ L'époque est à la perception des formes, à l'expérience du sensible, à la critique de la raison:

La pensée adulte, normale et civilisée vaut mieux que la pensée enfantine, morbide ou barbare mais a une condition, c'est qu'elle ne se prenne pas pour pensée de droit divin, qu'elle se mesure toujours plus honnêtement aux obscurités et aux difficultés de la vie humaine, qu'elle ne perde pas le contact avec les racines irrationnelles de cette vie et qu'enfin la raison reconnaisse que son monde aussi est inachevé, ne feigne pas d'avoir dépassé ce qu'elle s'est bornée à masquer et ne prenne pas pour incontestables une civilisation et une connaissance que sa fonction la plus haute est au contraire de contester. ${ }^{45}$

Le cas Sandorama rend compte de l'importance des revues pharmaceutiques suisses de la seconde moitié du $\mathrm{XX}^{\mathrm{e}}$ siècle dans l'appréhension des relations entre médecine, arts et commerce. Cette archive permet de documenter, dans le cadre franco-suisse, une période mal connue des relations entre arts et médecine qui se caractérise par un rapport surdéterminé, de part et

41 Pic 2018a.

42 Derrida 1968.

43 Freud 1904.

44 Hersch 1981.

45 Merleau-Ponty 1948, IV $\$ 5$. 
d'autre, à l'expérimentation scientifique et formelle. Le constat est en effet évident d'une rupture entre l'humanisme des revues médicales des années 1930 et celui des années 1960, le second recourant expérimentalement aux procédures artistiques au lieu de s'établir sur les fondements moraux et esthétiques du classicisme. L'énonciation, la figure du lecteur, l'humour, l'inédit et le montage sont autant de modalités d'un rapport expérimental entre les arts et la littérature dans Sandorama. Leur analyse à partir des pratiques énonciatives et visuelles de la revue permet de saisir les enjeux socio-culturels de la publicité pharmaceutique et du film scientifique: produire une relation de confiance par les formes qui, dans les années 1960, tient ensemble arts et médecine au sein du dispositif économique de l'industrie pharmaceutique en pleine révolution psychopharmacologique. L'analyse des revues montre donc le crédit réciproque que se donnent arts et sciences. Une archive comme Sandorama invite à s'interroger sur ce qu'humanisme veut dire à cette époque, mais aussi à la nôtre où s'impose le concept d'humanités médicales. Pour y répondre, il faudrait probablement le situer dans le contexte plus large de l'histoire de la raison et de ses formes.

\section{Bibliographie}

Aron, Paul/Benoît Denis, «Réseaux et institution faible», in: Daphné de Marneffe/ Benoît Denis (éds), Les Réseaux littéraires (Bruxelles 2006) 7-18

Aron, Paul, «Les revues littéraires: histoire et problématique», COnTEXTES 4 (2008) mis en ligne le 28 octobre 2008 http://journals.openedition.org/contextes/3813, consulté le 2 mars 2020

Aron, Paul, «La poésie professionnelle des pharmaciens au XIX ${ }^{\mathrm{e}}$ et $\mathrm{XX}^{\mathrm{e}}$ siècles en France et en Belgique» in: Thomas Augais/Martina Diaz/Julien Kneubusch/Alexandre Wenger (éds.), La figure du poète-médecin, 20e-21e siècles (Genève 2018) 173-196

Barras, Vincent, «Organe» in: Lydie Bodiou et al. (éds), Dictionnaire du corps dans l'Antiquité (Rennes 2019) 454-455

Bonah, Christian/Joël Danet, «L'expérience 'Medfilm': usages des archives du film utilitaire comme outils pédagogiques, ou 'objets (pré) transitionnels', dans le cadre de l'enseignement des Humanités médicales», Tréma 48 (2018) 35-50 http://journals.openedition.org/trema/3862, mis en ligne le 1 juin 2018, consulté le 9 février 2020

Campanelli, Alessandro, L'émergence de l'État helvétique entre unité et fédéralisme: l'exemple des législations médicales et pharmaceutiques (1798-1900) (Genève 2018)

Derrida, Jacques, «La pharmacie de Platon» [1968], in: La Dissémination (Paris 1972)

Dettwiler, Walter (éd.), 15 Jahre Novartis. 150 Jahre Innovation (Bâle 2011) 
Díaz, Martina, «Esthétiser la médecine: la revue Art et Médecine (1929-1939)», Cahiers Internationaux du symbolisme 146-147-148 (2017) 75-92

Díaz, Martina, «Panorama des revues médico-littéraires de l'entre-deux-guerres», Epistémocritique. Réseaux médico-littéraires dans l'Entre-deux-guerres: revues, institutions, lieux, figures (septembre 2018) 15-45

http://epistemocritique.org/panorama-des-revues-medico-litteraires-a-lentredeux-guerres/, consulté le 20 décembre 2019

Díaz, Martina/Alexandre Wenger, «Henri Mondor et la revue Art et Médecine: construction de l'idéal du médecin-littérateur dans les années 1930», in: Julia Pröll/ Hans-Jürgen Lüsebrink (éds), Médecins-écrivains français et francophones (Würzburg 2018) 99-116

Ducrot, Oswald, Le dire et le dit (Paris 1984)

Edel, Yves, «Expérimentations des psychédéliques à Saint-Anne dans les années 1960», Annales Médico-psychologiques 175 (2017) 653-660

Eikhenbaum, Boris, «Problèmes de ciné-stylistique» (1927), trad. par Sylviane Mossé et André Robel, in: François Albera (éd.), Les formalistes russes et le cinéma. Poétique du film (Paris 1996)

Foucault, Michel, Surveiller et punir (Paris 1975)

Freud, Sigmund, Le Mot d'esprit et sa relation à l'inconscient [1904], trad. Denis Messier (Paris 1988)

Gaudillière, Jean-Paul, «Marketing loop: Clinical Research, Consumption of Antidepressants and the Reorganization of Promotion at Geigy in the 1960s and 1970s», in: Jean-Paul Gaudilliere/Ulrike Thoms (éds), The Development of Scientific Marketing in the Twentieth Century, = Studies for the Society for the Social History of Medecine 22 (2015) 167-189

Genette, Gérard, «Morts de rire», Figure V (Paris 2002)

Guellec, Laurence, «Le commerce de la science: poésie scientifique et rhétorique publicitaire», in: Muriel Louâpre/Hugues Marchal/Michel Pierssens (éds), La poésie scientifique, de la gloire au déclin, actes du colloque de Montréal, 15-17 septembre 2010, Épistémocritique

http://epistemocritique.org/category/ouvrages-en-ligne/actes-de-colloques/lapoesie-scientifique-de-la-gloire-au-declin/, consulté le 20 décembre 2019

Guellec, Laurence, «Les poètes publicitaires et la tradition de la réclame en vers au $\mathrm{XIX}^{\mathrm{e}}$ siècle», in: Marie-Paule Berranger/Laurence Guellec (éds), Les Poètes et la publicité. Actes des journées d'études des 15 et 16 janvier 2016 (Paris 2017) 46-78 http://littepub.net /publication/je-poetes-publicite/l-guellec.pdf, consulté le 20 décembre 2019

Guellec, Laurence/Françoise Hache-Bissette (éds), Littérature et publicité. De Balzac à Beigbeder: actes du colloque international des Arts décoratifs (28-30 avril 2011) (Marseille 2012)

Hersch, Jeanne, L'étonnement philosophique (Paris 1981)

Jansers, Andres, «Design as Giving: Graphic design and Advertising from Basel», in: Andres Jansers/Barbara Junod (éds.), Corporate Diversity. Swiss Graphic Design and Advertising by Geigy. 1940-1970 (Zurich 2009) 8-29

Junod, Barbara, «From a Focus on Products to a Focus on Customers - The Advertising Policies and Practices at the Basel Headquarters», in: Andres Jansers/Bar- 
bara Junod (éds), Corporate Diversity. Swiss Graphic Design and Advertising by Geigy. 1940-1970 (Zurich 2009) 30-47

Jolles, André, Formes simples [1930], trad. par Antoine Marie Buguet (Paris 1972)

Kuhn, Thomas S., La Structure des révolutions scientifiques [1970] (Paris 2018)

Lefebvre, Thierry, «Le film médical au $\mathrm{XX}^{\mathrm{e}}$ siècle. Le cinéma au service de la médecine et des médecins», in: Florence Douguet et al. (éds), Image et santé (Paris 2011) 135-147

Lefebvre, Thierry, «L'épopée de la cinémathèque Sandoz», Revue d'histoire de la pharmacie 62 (2014) 393-404

Lempérière, Thérèse, «Hommage à Jean Delay», in: Florence Delay (éd.), Hommage à Jean Delay (Paris 2008)

Leibovici, Franck, Henri Michaux: Voir (Une enquête) (Paris 2014)

Maldonaldo, Guitemie, Le Cercle et l'amibe: le biomorphisme dans l'art des années 1930 (Paris 2006)

Marchal, Hughes (éd.), Muses et ptérodactyles. La poésie de la science de Chénier à Rimbaud (Paris 2013)

Marchal, Hugues, «Chassés-croisés. La littérature au miroir des revues scientifiques», in: Tania Collani/Noëlle Cuny (éds), Poétiques scientifiques dans les revues européennes de la modernité (1900-1940) (Paris 2015)

Marchal, Hughes, «La poésie des carabins: l'Anthologie hospitalière et latinesque de Courtepaille», in: Thomas Augais/Martina Diaz/Julien Knebusch/Alexandre Wenger (éds), La figure du poète-médecin, XX'e-XXI' siècles (Genève 2018) 197-218

Merleau-Ponty, Maurice, Phénoménologie de la perception [1945], in: Euvres, éd. par Claude Lefort (Paris 2010)

Merleau-Ponty, Maurice, Causeries [1948] (Paris 2002)

Neuburg, Hans, Chemie, Werbung und Graphik. Publicité et graphisme dans l'industrie chimique, avec la collaboration de René Rudin, Victor N. Cohen et Josef Müller-Brockmann (Zurich 1967)

Portmann, Adolf, La Forme animale (1948), trad. par Georges Rémy (Paris 2013)

Pic, Muriel (éd.), Edith Boissonnas et Jean Dubuffet. La Vie est libre. Correspondance et documents 1945-1980 (Genève 2014a)

Pic, Muriel (éd. et préf.), Mescaline 55, Edith Boissonnas, Henri Michaux, Jean Paulhan (Paris 2014b)

Pic, Muriel, «Par la voie des nerfs. Henri Michaux et l'usage clinique des psychotropes», Mouvement, $\mathrm{n}^{\circ}$ spécial Ordres et désordres des drogues (2016a) 142-150

Pic. Muriel, «Témoin, qu'as-tu fait de tes yeux? Henri Michaux et l'auto-observation», Hippocampe 13 (2016b) 5-16

Pic, Muriel, «Qu'est-ce que s'orienter dans les images?», Europe, $\mathrm{n}^{\circ}$ spécial Georges Didi-Huberman, 1069 (2018a) 3-17

Pic, Muriel, «Archives animales», Artpress2 48, Laurent Perez (éd.), «L'animal notre histoire» (2018b) 86-91

https://www.artpress.com/2018/04/26/artpress2-n48-lanimal-notre-histoire/, consulté le 20 décembre 2019

Pigeaud, Jackie, La Maladie de l'âme, Étude sur la relation de l'âme et du corps dans la tradition médico-philosophique antique (Paris 1981)

Rosenthal, Victor/Yves-Marie Visetti, «Sens et temps de la Gestalt», Intellectica 28 (1999) 147-227 
https://formes-symboliques.org/IMG/pdf/doc-79.pdf, consulté le 20 décembre 2019

Starobinski, Jean, «Introduction», in: Hans Prinzhorn. Expressions de la folie: dessins, peintures, sculptures d'asile, éd. par Marielène Weber, trad. par Alain Brousse et Marielène Weber (Paris 1984)

Starobinski, Jean, «Plaidoyer pour des humanités médicales», in: Gérard Danou (éd.), Littérature et médecine, ou Les pouvoirs du récit (Paris 2001) 7-8

Suter, Patrick, Le journal et les Lettres. 1. De la presse à l'œuvre (Mallarmé-Futurisme - Dada - Surréalisme) (Genève 2010)

Suter, Patrick, Le journal et les Lettres. 2. La presse dans l'œuvre: vers une écologie littéraire (Butor, Simon, Rolin) (Genève 2010)

Suter, Patrick, «Publicité et poésie visuelle: du poème-affiche aux poèmes lumineux», in: Isabelle Chol/Bénédicte Mathios/Serge Linarès (éds), LiVres de pOésie. Jeux d'eSpace (Paris 2016) 183-204 